CALT-68-2896

\title{
SPECTRAL CURVES AND THE SCHRÖDINGER EQUATIONS FOR THE EYNARD-ORANTIN RECURSION
}

\author{
MOTOHICO MULASE AND PIOTR SUŁKOWSKI
}

\begin{abstract}
It is predicted that the principal specialization of the partition function of a B-model topological string theory, that is mirror dual to an A-model enumerative geometry problem, satisfies a Schrödinger equation, and that the characteristic variety of the Schrödinger operator gives the spectral curve of the B-model theory, when an algebraic K-theory obstruction vanishes. In this paper we present two concrete mathematical A-model examples whose mirror dual partners exhibit these predicted features on the B-model side. The A-model examples we discuss are the generalized Catalan numbers of an arbitrary genus and the single Hurwitz numbers. In each case, we show that the Laplace transform of the counting functions satisfies the Eynard-Orantin topological recursion, that the B-model partition function satisfies the KP equations, and that the principal specialization of the partition function satisfies a Schrödinger equation whose total symbol is exactly the Lagrangian immersion of the spectral curve of the Eynard-Orantin theory.
\end{abstract}

\section{Contents}

1. Introduction and the main results

2. The Eynard-Orantin topological recursion

3. The generalized Catalan numbers and the topological recursion

4. The partition function for the generalized Catalan numbers and the Schrödinger equation

5. Single Hurwitz numbers

6. The Schur function expansion of the Hurwitz partition function 24

7. Conclusion

Appendix A. Proof of the Schrödinger equation for the Catalan case 31

Appendix B. Hierarchy of equations for $S_{m}$

References

\section{INTRODUCTION AND THE MAIN RESUltS}

In a series of remarkable papers of Mariño [51] and Bouchard, Klemm, Mariño, and Pasquetti [8], these authors have developed an inductive mechanism to calculate a variety of quantum invariants and solutions to enumerative geometry questions, based on the fundamental work of Eynard and Orantin [27, 28, 29]. The validity of their method, known as the remodeled B-model based on the topological recursion of Eynard-Orantin, has been established for many different enumerative geometry problems, such as single Hurwitz numbers ([7, 26, 60], based on the conjecture of Bouchard and Mariño [9]), open Gromov-Witten invariants of smooth toric Calabi-Yau threefolds ([29, 80, based on the remodeling conjecture of Mariño [51] and Bouchard, Klemm, Mariño, Pasquetti [8]), and the number of lattice

2000 Mathematics Subject Classification. Primary: 14H15, 14N35, 05C30, 11P21; Secondary: 81T30. 
points on $\mathcal{M}_{g, n}$ and its symplectic and Euclidean volumes ([11, 20, 58, based on 63, 64]). It is expected that double Hurwitz numbers, stationary Gromov-Witten invariants of $\mathbb{P}^{1}$ 65, 66], certain Donaldson-Thomas invariants, and many other quantum invariants would also fall into this category.

Unlike the familiar Topological Recursion Relations (TRR) of the Gromov-Witten theory, the Eynard-Orantin recursion is a B-model formula [8, 51]. The significant feature of the formula is its universality: independent of the A-model problem, the B-model recursion takes always the same form. The input data of this B-model consist of a holomorphic Lagrangian immersion

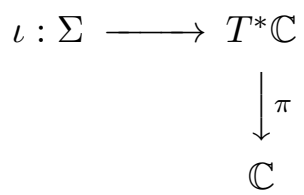

of an open Riemann surface $\Sigma$ (called a spectral curve of the Eynard-Orantin recursion) into the cotangent bundle $T^{*} \mathbb{C}$ equipped with the tautological 1-form $\eta$, and the symmetric second derivative of the logarithm of Riemann's prime form [30, 62] defined on $\Sigma \times \Sigma$. The procedure of Eynard-Orantin [27] then defines, inductively on $2 g-2+n$, a meromorphic symmetric differential $n$-form $W_{g, n}$ on $\Sigma^{n}$ for every $g \geq 0$ and $n \geq 1$ subject to $2 g-2+n>0$. A particular choice of the Lagrangian immersion gives a different $W_{g, n}$, which then gives a generating function of the solution to a different enumerative geometry problem.

Thus the real question is how to find the right Lagrangian immersion from a given Amodel.

Suppose we have a solution to an enumerative geometry problem (an A-model problem). Then we know a generating function of these quantities. In [20] we proposed an idea of identifying the spectral curve $\Sigma$, which states that the spectral curve is the Laplace transform of the disc amplitude of the A-model problem. Here the Laplace transform plays the role of mirror symmetry. Thus we obtain a Riemann surface $\Sigma$. Still we do not see the aspect of the Lagrangian immersion in this manner.

Every curve in $T^{*} \mathbb{C}$ is trivially a Lagrangian. But not every Lagrangian is realized as the mirror dual to an A-model problem. The obstruction seems to lie in the K-group $K_{2}(\mathbb{C}(\Sigma)) \otimes \mathbb{Q}$. When this obstruction vanishes, we call $\Sigma$ a $K_{2}$-Lagrangian, following Kontsevich's terminology. For a $K_{2}$-Lagrangian $\Sigma$, we expect the existence of a holonomic system that characterizes the partition function of the B-model theory, and at the same time, the characteristic variety of this holonomic system recovers the spectral curve $\Sigma$ as the Lagrangian immersion. A generator of this holonomic system is called a quantum Riemann surface [1, 16, 17], because it is a differential operator whose total symbol is the spectral curve realized as a Lagrangian immersion [18. It is the work of Gukov and Sułkowski [39] that suggested the obstruction to the existence of the holonomic system with algebraic K-theory as an element of $K_{2}$.

Another mysterious link of the Eynard-Orantin theory is its relation to integrable systems of the $\mathrm{KP} / \mathrm{KdV}$ type [5, 27. We note that the partition function of the B-model is always the principal specialization of a $\tau$-function of the KP equations for all the examples we know by now.

The purpose of the present paper is to give the simplest non-trivial mathematical examples of the theory that exhibit these key features mentioned above. With these examples one can calculate all quantities involved, give proofs of the statements predicted in physics, and examine the mathematical structure of the theory. Our examples are based on enumeration problems of branched coverings of $\mathbb{P}^{1}$. 
The idea of homological mirror symmetry of Kontsevich [44] allows us to talk about the mirror symmetry without underlying spaces, because the formulation is based on the derived equivalence of categories. Therefore, we can consider the mirror dual B-models corresponding to the enumeration problems of branched coverings on the A-model side. At the same time, being the derived equivalence, the homological mirror symmetry does not tell us any direct relations between the quantum invariants on the A-model side and the complex geometry on the B-model side. This is exactly where Mariño's idea of remodeling $\mathrm{B}$-model comes in for rescue. The remodeled B-model of [8, 51] is not a derived category of coherent sheaves. Although its applicability is restricted to the case when there is a family of curves $\Sigma$ that exhibits the geometry of the B-model, the new idea is to construct a network of inter-related differential forms on the symmetric powers of $\Sigma$ via the EynardOrantin recursion, and to understand this infinite system as the B-model. The advantage of this idea is that we can relate the solution of the geometric enumeration problem on the A-model side and the symmetric differential forms on the B-model side through the Laplace transform. In this sense we consider the Laplace transform as a mirror symmetry.

The first example we consider in this paper is the generalized Catalan numbers of an arbitrary genus. This is equivalent to the " $c=1$ model" of [39, Section 5]. In terms of enumeration, we are counting the number of algebraic curves defined over $\overline{\mathbb{Q}}$ in a systematic way by using the dual graph of Grothendieck's dessins d'enfants [4, 74.

Let $D_{g, n}\left(\mu_{1}, \ldots, \mu_{n}\right)$ denote the automorphism-weighted count of the number of connected cellular graphs on a closed oriented surface of genus $g$ (i.e., the 1-skeleton of celldecompositions of the surface), with $n$ labeled vertices of degrees $\left(\mu_{1}, \ldots, \mu_{n}\right)$. The letter D stands for 'dessin.' The generalized Catalan numbers of type $(g, n)$ are defined by

$$
C_{g, n}\left(\mu_{1}, \ldots, \mu_{n}\right)=\mu_{1} \cdots \mu_{n} D_{g, n}\left(\mu_{1}, \ldots, \mu_{n}\right) .
$$

While $D_{g, n}(\vec{\mu})$ is a rational number due to the graph automorphisms, the generalized Catalan number $C_{g, n}(\vec{\mu})$ is always a non-negative integer. It gives the dimension of a linear skein space. In particular, the $(g, n)=(0,1)$ case recovers the original Catalan numbers:

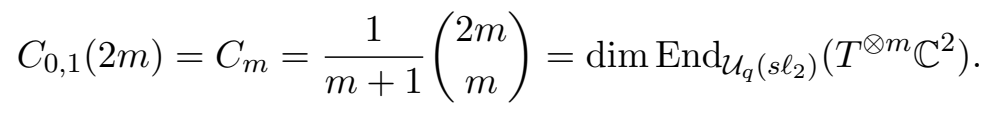

As explained in [20], the mirror dual to the Catalan numbers $C_{m}$ is the plane curve $\Sigma$ defined by

$$
\left\{\begin{array}{l}
x=z+\frac{1}{z} \\
y=-z
\end{array}\right.
$$

where

$$
z(x)=\sum_{m=0}^{\infty} C_{m} \frac{1}{x^{2 m+1}}
$$

Note that (1.1) also gives a Lagrangian immersion $\Sigma \longrightarrow T^{*} \mathbb{C}$. Let us introduce the free energies by

$$
F_{g, n}^{C}\left(z\left(x_{1}\right), \ldots, z\left(x_{n}\right)\right)=\sum_{\vec{\mu} \in \mathbb{Z}_{+}^{n}} D_{g, n}(\vec{\mu}) e^{-\left(w_{1} \mu_{1}+\cdots+w_{n} \mu_{n}\right)}=\sum_{\vec{\mu} \in \mathbb{Z}_{+}^{n}} D_{g, n}(\vec{\mu}) \prod_{i=1}^{n} \frac{1}{x_{i}^{\mu_{i}}}
$$

as the Laplace transform of the number of dessins, where the coordinates are related by 1.2 and $x_{i}=e^{w_{i}}$. The free energy $F_{g, n}^{C}\left(z_{1}, \ldots, z_{n}\right)$ is a symmetric function in $n$-variables, 
and its principal specialization is defined by $F_{g, n}^{C}(z, \ldots, z)$. Now let

$$
W_{g, n}^{C}\left(z_{1}, \ldots, z_{n}\right)=d_{1} \cdots d_{n} F_{g, n}\left(z_{1}, \ldots, z_{n}\right) .
$$

It is proved in [20] that $W_{g, n}^{C}$ 's satisfy the Eynard-Orantin topological recursion.

The Catalan partition function is given by the formula of [27]:

$$
Z^{C}(z, \hbar)=\exp \left(\sum_{g=0}^{\infty} \sum_{n=1}^{\infty} \frac{1}{n !} \hbar^{2 g-2+n} F_{g, n}^{C}(z, z, \ldots, z)\right) .
$$

In this paper we prove

Theorem 1.1. The Catalan partition function satisfies the Schrödinger equation

$$
\left(\hbar^{2} \frac{d^{2}}{d x^{2}}+\hbar x \frac{d}{d x}+1\right) Z^{C}(z(x), \hbar)=0 .
$$

The characteristic variety of this ordinary differential operator, $y^{2}+x y+1=0$ for every fixed choice of $\hbar$, is exactly the Lagrangian immersion (1.1), where we identify the $x y$-plane as the cotangent bundle $T^{*} \mathbb{C}$ with the fiber coordinate $y=\hbar \frac{d}{d x}$.

Remark 1.2. A purely geometric reason of our interest in the function appearing as the principal specialization $F_{g, n}^{C}(z, \ldots, z)$ is that, in the stable range $2 g-2+n>0$, it is a polynomial in

$$
s=\frac{z^{2}}{z^{2}-1}
$$

of degree $6 g-6+3 n$. It is indeed the virtual Poincaré polynomial of $\mathcal{M}_{g, n} \times \mathbb{R}_{+}^{n}$ [58], and its special value at $s=1$ gives the Euler characteristic $(-1)^{n} \chi\left(\mathcal{M}_{g, n}\right)$ of the moduli space $\mathcal{M}_{g, n}$ of smooth $n$-pointed curves of genus $g$. Thus $Z^{C}(z, \hbar)$ is the exponential generating function of the virtual Poincaré polynomials of $\mathcal{M}_{g, n} \times \mathbb{R}_{+}^{n}$.

As such, the generating function $Z^{C}(z, \hbar)$ is also expressible in terms of a Hermitian matrix integral

$$
Z^{C}(z, \hbar)=\int_{\mathcal{H}_{N \times N}} \operatorname{det}(1-\sqrt{s} X)^{N} e^{-\frac{N}{2} \operatorname{trace}\left(X^{2}\right)} d X
$$

with the identification (1.6) and $\hbar=1 / N$. Here $d X$ is the normalized Lebesgue measure on the space of $N \times N$ Hermitian matrices $\mathcal{H}_{N \times N}$. It is a well-known fact that Eq. (1.7) is the principal specialization of a KP $\tau$-function (see for example, [54]).

Another example we consider in this paper is based on single Hurwitz numbers. As a counting problem it is easier to state than the previous example, but the Lagrangian immersion requires a transcendental function, and hence the resulting Schrödinger equation exhibits a rather different nature.

Let $H_{g, n}\left(\mu_{1} \ldots, \mu_{n}\right)$ be the automorphism-weighted count of the number of topological types of Hurwitz covers $f: C \longrightarrow \mathbb{P}^{1}$ of a connected non-singular algebraic curve $C$ of genus g. A holomorphic map $f$ is a Hurwitz cover of profile $\left(\mu_{1} \ldots, \mu_{n}\right)$ if it has $n$ labeled poles of orders $\left(\mu_{1} \ldots, \mu_{n}\right)$ and is simply ramified otherwise. Introduce the Laplace transform of single Hurwitz numbers by

$$
F_{g, n}^{H}\left(t\left(w_{1}\right), \ldots, t\left(w_{n}\right)\right)=\sum_{\vec{\mu} \in \mathbb{Z}_{+}^{n}} H_{g, n}(\vec{\mu}) e^{-\left(w_{1} \mu_{1}+\cdots w_{n} \mu_{n}\right)},
$$


where

$$
t(w)=\sum_{m=0}^{\infty} \frac{m^{m}}{m !} e^{-m w}
$$

is the tree-function. Here again $F_{g, n}^{H}\left(t_{1}, \ldots, t_{n}\right)$ is a polynomial of degree $6 g-6+3 n$ if $2 g-2+n>0$ [60]. Bouchard and Mariño have conjectured [9] that

$$
W_{g, n}^{H}\left(t_{1}, \ldots, t_{n}\right)=d_{1} \cdots d_{n} F_{g, n}^{H}\left(t_{1}, \ldots, t_{n}\right)
$$

satisfy the Eynard-Orantin topological recursion, with respect to the Lagrangian immersion

$$
\left\{\begin{array}{l}
x=e^{-w}=z e^{-z} \in \mathbb{C}^{*} \\
y=z \in \mathbb{C}
\end{array}\right.
$$

where

$$
z=\frac{t-1}{t}
$$

and we use $\eta=y \frac{d x}{x}$ as the tautological 1-form on $T^{*} \mathbb{C}^{*}$. The Bouchard-Mariño conjecture was proved in [7, 26, 60].

Now we define the Hurwitz partition function

$$
Z^{H}(t, \hbar)=\exp \left(\sum_{g=0}^{\infty} \sum_{n=1}^{\infty} \frac{1}{n !} \hbar^{2 g-2+n} F_{g, n}^{H}(t, \ldots, t)\right) .
$$

Then we have the following

Theorem 1.3. The Hurwitz partition function satisfy two equations:

$$
\begin{aligned}
& {\left[\frac{1}{2} \hbar \frac{\partial^{2}}{\partial w^{2}}+\left(1+\frac{1}{2} \hbar\right) \frac{\partial}{\partial w}-\hbar \frac{\partial}{\partial \hbar}\right] Z^{H}(t(w), \hbar)=0,} \\
& \left(\hbar \frac{d}{d w}+e^{-w} e^{-\hbar \frac{d}{d w}}\right) Z^{H}(t(w), \hbar)=0 .
\end{aligned}
$$

Moreover, each of the two equations recover the Lagrangian immersion (1.9) from the asymptotic analysis at $\hbar \sim 0$. And if we view $\hbar$ as a fixed constant in $(1.12)$, then its total symbol is the Lagrangian immersion (1.9) with the identification $z=-\hbar \frac{d}{d w}$.

Remark 1.4. The second order equation (1.11) is a consequence of the polynomial recursion of [60]. This situation is exactly the same as Theorem 1.1. The differential-difference equation 1.12, or a delay differential equation, follows from the principal specialization of the KP $\tau$-function that gives another generating function of single Hurwitz numbers [41, 67. We remark that (1.12) is also derived in [82]. The point of view of differential-difference equation is further developed in [59] for the case of double Hurwitz numbers and $r$-spin structures, where we generalize a result of 82 .

Remark 1.5. Define two operators by

$$
\begin{aligned}
& P=\hbar \frac{d}{d w}+e^{-w} e^{-\hbar \frac{d}{d w}} \quad \text { and } \\
& Q=\frac{1}{2} \hbar \frac{\partial^{2}}{\partial w^{2}}+\left(1+\frac{1}{2} \hbar\right) \frac{\partial}{\partial w}-\hbar \frac{\partial}{\partial \hbar} .
\end{aligned}
$$

Then it is noted in [49] that

$$
[P, Q]=P
$$


Thus the heat equation (1.11) preserves the space of solutions of the Schrödinger equation (1.12). In this sense, $(1.12)$ is holonomic for every fixed $\hbar$. The analysis of these equations is further investigated in [49].

The existence of a holonomic system is particularly appealing when we consider the knot A-polynomial as the defining equation of a Lagrangian immersion, in connection to the AJ conjecture [33, 34, 38]. One can ask:

Question 1.6. Let $K$ be a knot in $S^{3}$ and $A_{K}$ its A-polynomial [13]. Is there a concrete formula for the quantum knot invariants of $K$, such as the colored Jones polynomials, in terms of $A_{K}$ ?

The B-model developed in [15], 39], and more recently in [6], clearly shows that the answer is yes, and it should be given by the Eynard-Orantin formalism. Although our examples in the present paper are not related to any knots, they suggest the existence of a corresponding A-model. An interesting theory of generalized A-polynomials and quantum knot invariants from the point of view of mirror symmetry is recently presented in Aganagic and Vafa [2. We also remark that there are further developments in this direction [10, 31, 32 .

The paper is organized as follows. In Section 2, we give the definition of the EynardOrantin topological recursion. We emphasize the aspect of Lagrangian immersion in our presentation. In Section 3 we review the generalized Catalan numbers of [20]. Then in Section 4 we derive the Schrödinger equation for the Catalan partition function. The equation for the Hurwitz partition function is given in Section 5 . Finally, in Section 66 we give the proof of (1.12) using the Schur function expansion of the Hurwitz generating function and its principal specialization.

\section{ThE EynARD-ORANTIN TOPOLOGICAL RECURSION}

We adopt the following definition for the topological recursion of Eynard-Orantin [27. Our emphasis, which is different from the original, is the point of view of the Lagrangian immersion.

Definition 2.1. The spectral curve $(\Sigma, \iota)$ consists of an open Riemann surface $\Sigma$ and a Lagrangian immersion

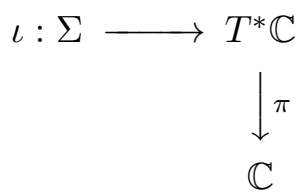

with respect to the canonical holomorphic symplectic structure $\omega=-d \eta$ on $T^{*} \mathbb{C}$, where $\eta$ is the tautological 1-form on the cotangent bundle $\pi: T^{*} \mathbb{C} \longrightarrow \mathbb{C}$. Recall that $p \in \Sigma$ is a Lagrangian singularity if $d(\pi \circ \iota)=0$ at $p$, and that $\pi(\iota(p)) \in \mathbb{C}$ is a caustic of the Lagrangian immersion. We assume that the projection $\pi$ restricted to the Lagrangian immersion is locally simply ramified around each Lagrangian singularity. We denote by $R=\left\{p_{1}, \ldots, p_{r}\right\} \subset \Sigma$ the set of Lagrangian singularities, and by

$$
U=\sqcup_{j=1}^{r} U_{j}
$$

the disjoint union of small neighborhood $U_{j}$ around $p_{j}$ such that $\pi: U_{j} \longrightarrow \pi\left(U_{j}\right) \subset \mathbb{C}$ is a double-sheeted covering ramified only at $p_{j}$. We denote by $s_{j}(z)$ the local Galois conjugate of $z \in U_{j}$. 
Another key ingredient of the Eynard-Orantin theory is the normalized fundamental differential of the second kind $B_{\Sigma}\left(z_{1}, z_{2}\right)$ [30, Page 20], 62, Page 3.213], which is a symmetric differential 2 -form on $\Sigma \times \Sigma$ with second-order poles along the diagonal. To define it, let us recall a few basic facts about algebraic curves. Let $C$ be a nonsingular complete algebraic curve over $\mathbb{C}$. We are considering a nonsingular compactification $C=\bar{\Sigma}$ of the Riemann surface $\Sigma$. We identify the Jacobian variety of $C$ as $\operatorname{Jac}(C)=\operatorname{Pic}^{0}(C)$, which is isomorphic to $\mathrm{Pic}^{g-1}(C)$. The theta divisor $\Theta$ of $\mathrm{Pic}^{g-1}(C)$ is defined by

$$
\Theta=\left\{L \in \operatorname{Pic}^{g-1}(C) \mid \operatorname{dim} H^{1}(C, L)>0\right\} .
$$

We use the same notation for the translate divisor on $\operatorname{Jac}(C)$, also called the theta divisor. Now consider the diagram

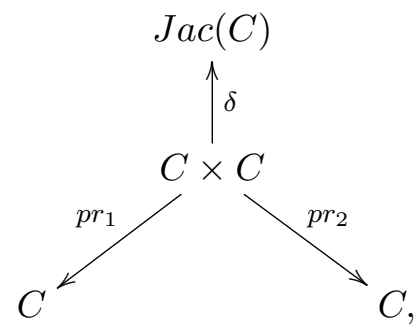

where $p r_{j}$ denotes the projection to the $j$-th components, and

$$
\delta: C \times C \ni(p, q) \longmapsto p-q \in J a c(C) .
$$

Then the prime form $E_{C}\left(z_{1}, z_{2}\right)$ [30, Page 16] is defined as a holomorphic section

$$
E_{C}(p, q) \in H^{0}\left(C \times C, p r_{1}^{*} K_{C}^{-\frac{1}{2}} \otimes p r_{2}^{*} K_{C}^{-\frac{1}{2}} \otimes \delta^{*}(\Theta)\right),
$$

where $K_{C}$ is the canonical line bundle of $C$ and we choose Riemann's spin structure (or the Szegö kernel) $K_{C}^{\frac{1}{2}}$ (see [30, Theorem 1.1]). We do not need the precise definition of the prime form here, but its characteristic properties are important:

(1) $E_{C}(p, q)$ vanishes only along the diagonal $\Delta \subset C \times C$, and has simple zeros along $\Delta$.

(2) Let $z$ be a local coordinate on $C$ such that $d z(p)$ gives the local trivialization of $K_{C}$ around $p$. When $q$ is near at $p, \delta^{*}(\Theta)$ is also trivialized around $(p, q) \in C \times C$, and we have a local behavior

$$
E_{C}(z(p), z(q))=\frac{z(p)-z(q)}{\sqrt{d z(p)} \cdot \sqrt{d z(q)}}\left(1+O\left((z(p)-z(q))^{2}\right)\right) .
$$

(3) $E_{C}(z(p), z(q))=-E_{C}(z(q), z(p))$.

The fundamental 2-form $B_{C}(p, q)$ is then defined by

$$
B_{C}(p, q)=d_{1} \otimes d_{2} \log E_{C}(p, q)
$$

(aee [30, Page 20], 62, Page 3.213]). We note that $d z(p)$ appears in (2.2) just as the indicator of our choice of the local trivialization. With this local trivialization, the square

$$
E_{C}(p, q)^{2} \in H^{0}\left(C \times C, p r_{1}^{*} K_{C}^{-1} \otimes p r_{2}^{*} K_{C}^{-1} \otimes \delta^{*}(\Theta)^{\otimes 2}\right)
$$

behaves better because of

$$
E_{C}(z(p), z(q))^{2}=\frac{(z(p)-z(q))^{2}}{d z(p) \cdot d z(q)}\left(1+O\left((z(p)-z(q))^{2}\right)\right) .
$$

We then see that 


$$
\begin{aligned}
B_{C}(z(p), z(q))=\frac{1}{2} d_{1} \otimes d_{2} \log E^{2}(z(p), z(q)) & \\
=\frac{d z(p) \cdot d z(q)}{(z(p)-z(q))^{2}}+O(1) d z(p) \cdot d z(q) & \quad \in H^{0}\left(C \times C, p r_{1}^{*} K_{C} \otimes p r_{2}^{*} K_{C} \otimes \mathcal{O}(2 \Delta)\right) .
\end{aligned}
$$

Definition 2.2. Let $D$ be a divisor of $\Sigma$. A meromorphic symmetric differential form of degree $n$ with poles along $D$ is an element of the symmetric tensor product

$$
\operatorname{Sym}^{n} H^{0}\left(\Sigma, K_{\Sigma}(* D)\right) \text {. }
$$

Definition 2.3. Meromorphic differential forms

$$
W_{g, n}\left(z_{1}, \ldots, z_{n}\right) \in \operatorname{Sym}^{n} H^{0}\left(\Sigma, K_{\Sigma}(* R)\right)
$$

for $g \geq 0$ and $n \geq 1$, subject to $2 g-2+n>0$, with poles along the Lagrangian singularities of the Lagrangian immersion $\Sigma \longrightarrow T^{*} \mathbb{C}$, are said to satisfy the Eynard-Orantin topological recursion if they satisfy the recursion formula

$$
\begin{aligned}
W_{g, n}\left(z_{1}, z_{2}, \ldots, z_{n}\right)=\frac{1}{2 \pi i} \sum_{j=1}^{r} \oint_{\gamma_{j}} & K_{j}\left(z, z_{1}\right)\left[W_{g-1, n+1}\left(z, s_{j}(z), z_{2}, \ldots, z_{n}\right)\right. \\
& \left.+\sum_{\substack{g_{1}+g_{2}=g \\
I \sqcup J=\{2,3, \ldots, n\}}}^{\text {No }(0,1) \text { terns }} W_{g_{1},|I|+1}\left(z, z_{I}\right) W_{g_{2},|J|+1}\left(s_{j}(z), z_{J}\right)\right] .
\end{aligned}
$$

Here the integration is taken with respect to $z \in U_{j}$ along a positively oriented simple closed loop $\gamma_{j}$ around $p_{j}$, and $z_{I}=\left(z_{i}\right)_{i \in I}$ for a subset $I \subset\{1,2, \ldots, n\}$. In the summation, "No $(0,1)$ terms" means the summand does not contain the terms with $g_{1}=0$ and $I=\emptyset$ or $g_{2}=0$ and $J=\emptyset$. The recursion kernels $K_{j}\left(z, z_{1}\right), j=1, \ldots, r$, are defined as follows. First we define $W_{0,1}$ and $W_{0,2}$.

$$
W_{0,1}(z)=\iota^{*} \eta=y d x \in H^{0}(\Sigma, K),
$$

where $x$ is a linear coordinate of $\mathbb{C}$ and $y$ is the dual coordinate of $T_{0}^{*} \mathbb{C}$ so that the Lagrangian immersion is given by

$$
\begin{gathered}
(x, y): \Sigma \ni t \longmapsto(x(z), y(z)) \in T^{*} \mathbb{C} . \\
W_{0,2}\left(z_{1}, z_{2}\right)=B_{\Sigma}\left(z_{1}, z_{2}\right)-\pi^{*} \frac{d x_{1} \cdot d x_{2}}{\left(x_{1}-x_{2}\right)^{2}} .
\end{gathered}
$$

We note that $W_{0,2}\left(z_{1}, z_{2}\right)$ is holomorphic along the diagonal $z_{1}=z_{2}$. The recursion kernel $K_{j}\left(z_{1}, z_{2}\right) \in H^{0}\left(U_{j} \times \Sigma, K_{U_{j}}^{-1} \otimes K_{\Sigma}\right)$ for $z_{1} \in U_{j}$ and $z_{2} \in \Sigma$ is defined by

$$
\begin{aligned}
K_{j}\left(z_{1}, z_{2}\right)=\frac{1}{2} \frac{1}{W_{0,1}\left(s_{j}\left(z_{1}\right)\right)-W_{0,1}\left(z_{1}\right)} & \otimes \int_{z_{1}}^{s_{j}\left(z_{1}\right)} W_{0,2}\left(\cdot, z_{2}\right) \\
= & \frac{1}{2} \frac{d_{2}\left(\log E_{\Sigma}\left(s_{j}\left(z_{1}\right), z_{2}\right)-\log E_{\Sigma}\left(z_{1}, z_{2}\right)\right)}{\left(y\left(s_{j}\left(z_{1}\right)\right)-y\left(z_{1}\right)\right) d x\left(z_{1}\right)} .
\end{aligned}
$$

The kernel $K_{j}\left(z_{1}, z_{2}\right)$ is an algebraic operator that multiplies $d z_{2}$ while contracts $\partial / \partial z_{1}$. 
The topological recursion is the reduction of $2 g-2+n$ by 1 , which is different from the usual boundary degeneration formula of $\overline{\mathcal{M}}_{g, n}$. As shown in Figure 2.1, the reduction corresponds to degeneration cycles of codimension 1 and 2, as in Arbarello-Cornalba-Griffiths [3. Chapter 17, Section 5, Page 589].

$$
\begin{aligned}
& \mathcal{M}_{0.3} \times \overline{\mathcal{M}}_{g, n-1} \longrightarrow \overline{\mathcal{M}}_{g, n}, \quad \mathcal{M}_{0.3} \times \overline{\mathcal{M}}_{g-1, n+1} \longrightarrow \overline{\mathcal{M}}_{g, n}, \\
& \mathcal{M}_{0.3} \times \bigcup_{\substack{g_{1}+g_{2}=g \\
n_{1}+n_{2}=n-1}} \overline{\mathcal{M}}_{g_{1}, n_{1}+1} \times \overline{\mathcal{M}}_{g_{2}, n_{2}+1} \longrightarrow \overline{\mathcal{M}}_{g, n}
\end{aligned}
$$
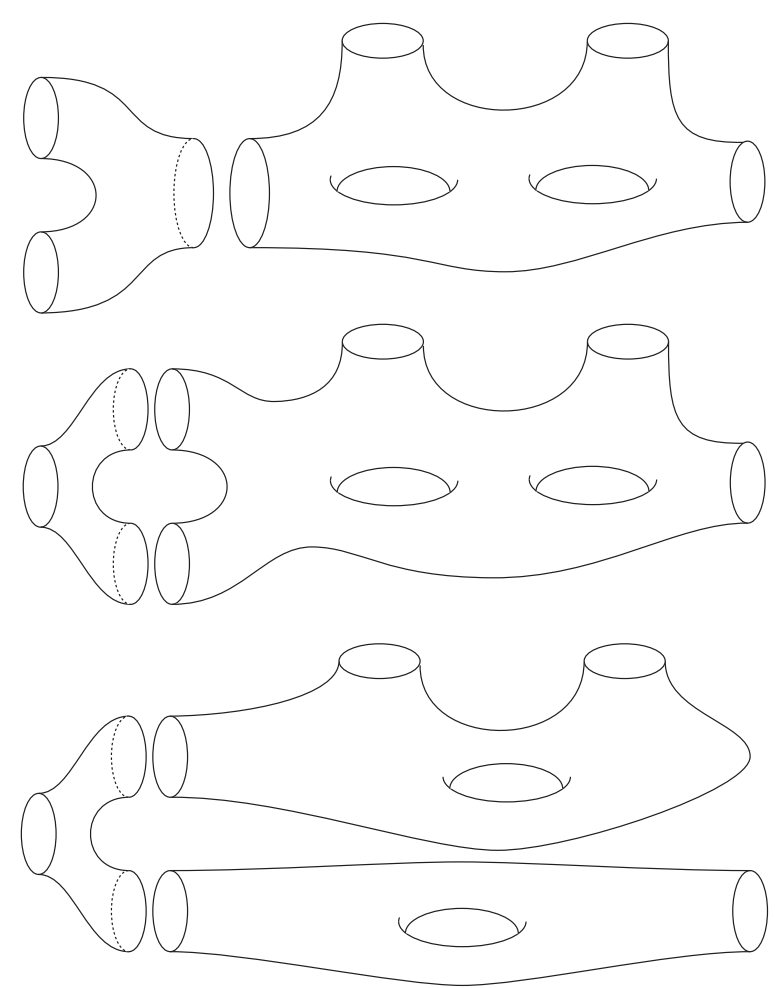

FiguRE 2.1. The topological recursion and degeneration.

The recursion starts from $W_{1,1}$ and $W_{0,3}$. If we modify (2.6) slightly, then these can also be calculated from $W_{0,2}[27]$.

$$
W_{1,1}\left(z_{1}\right)=\left.\frac{1}{2 \pi i} \sum_{j=1}^{r} \oint_{\gamma_{j}} K_{j}\left(z, z_{1}\right)\left[W_{0,2}(u, v)+c \pi^{*} \frac{d x(u) \cdot d x(v)}{(x(u)-x(v))^{2}}\right]\right|_{u=z, v=s_{j}(z)} .
$$

Formula (2.6) does not give an apparently symmetric expression for $W_{0,3}$. In terms of the coordinate $(x, y) \in T^{*} \mathbb{C}$ we have an alternative formula for $W_{0,3}$ [27]:

$$
W_{0,3}\left(z_{1}, z_{2}, z_{3}\right)=\frac{1}{2 \pi i} \sum_{j=1}^{r} \oint_{\gamma_{j}} \frac{W_{0,2}\left(z, z_{1}\right) W_{0,2}\left(z, z_{2}\right) W_{0,2}\left(z, z_{3}\right)}{d x(z) \cdot d y(z)} .
$$

Suppose we have a solution $W_{g, n}$ to the topological recursion. A primitive functions of the symmetric differential form $W_{g, n}$ is a symmetric meromorphic function $F_{g, n}$ on $\Sigma^{n}$ 
such that its $n$-fold exterior derivative recovers the $W_{g, n}$, i.e.,

$$
W_{g, n}\left(z_{1}, \ldots, z_{n}\right)=d_{z_{1}} \cdots d_{z_{n}} F_{g, n}\left(z_{1}, \ldots, z_{n}\right) .
$$

The partition function of the topological recursion for a genus 0 spectral curve is the formal expression in infinitely many variables

$$
Z\left(z_{1}, z_{2}, \ldots ; \hbar\right)=\exp \left(\sum_{g \geq 0, n \geq 1} \frac{1}{n !} \hbar^{2 g-2+n} F_{g, n}\left(z_{1}, z_{2}, \ldots, z_{n}\right)\right) .
$$

The principal specialization of the partition function is also denoted by the same letter $Z$ :

$$
Z(z, \hbar)=\exp \left(\sum_{g \geq 0, n \geq 1} \frac{1}{n !} \hbar^{2 g-2+n} F_{g, n}(z, z, \ldots, z)\right) .
$$

Remark 2.4. The partition function depends on the choice of the primitive functions. When we consider the topological recursion as the B-model corresponding to an A-model counting problem, then there is always a canonical choice for the primitives, as the Laplace transform of the quantum invariants.

Remark 2.5. When the spectral curve $\Sigma$ has a higher genus, the partition function requires a non-perturbative factor in terms of a theta function associated to the curve [5, 6]. In this case the algebraic K-theory condition of [39], probably similar to the Boutroux condition of [1], becomes essential for the existence of the quantum curve or the Schrödinger equation. Our consideration in paper is limited to the genus 0 case.

\section{The generalized Catalan numbers and the topological RECURSion}

A cellular graph of type $(g, n)$ is the one-skeleton of a cell-decomposition of a connected closed oriented surface of genus $g$ with $n 0$-cells labeled by the index set $[n]=\{1,2, \ldots, n\}$. Two cellular graphs are identified if an orientation-preserving homeomorphism of a surface into another surface maps one cellular graph to another, honoring the labeling of each vertex. Let $D_{g, n}\left(\mu_{1}, \ldots, \mu_{n}\right)$ denote the number of connected cellular graphs $\Gamma$ of type $(g, n)$ with $n$ labeled vertices of degrees $\left(\mu_{1}, \ldots, \mu_{n}\right)$, counted with the weight $1 /|\operatorname{Aut}(\Gamma)|$. It is generally a rational number. The orientation of the surface induces a cyclic order of incident half-edges at each vertex of a cellular graph $\Gamma$. Since $\operatorname{Aut}(\Gamma)$ fixes each vertex, the automorphism group is a subgroup of the Abelian group $\prod_{i=1}^{n} \mathbb{Z} / \mu_{i} \mathbb{Z}$ that rotates each vertex. Therefore,

$$
C_{g, n}\left(\mu_{1}, \ldots, \mu_{n}\right)=\mu_{1} \cdots \mu_{n} D_{g, n}\left(\mu_{1}, \ldots, \mu_{n}\right)
$$

is always an integer. The cellular graphs counted by (3.1) are connected graphs of genus $g$ with $n$ vertices of degrees $\left(\mu_{1}, \ldots, \mu_{n}\right)$, and at the $j$-th vertex for every $j=1, \ldots, n$, an outgoing arrow is placed on one of the incident $\mu_{j}$ half-edges (see Figure 3.1). The placement of $n$ arrows corresponds to the factors $\mu_{1} \cdots \mu_{n}$ on the right-hand side. We call this integer the generalized Catalan number of type $(g, n)$. The reason for this naming comes from the following theorem.

Theorem 3.1. The generalized Catalan numbers (3.1) satisfy the following equation.

$$
C_{g, n}\left(\mu_{1}, \ldots, \mu_{n}\right)=\sum_{j=2}^{n} \mu_{j} C_{g, n-1}\left(\mu_{1}+\mu_{j}-2, \mu_{2}, \ldots, \widehat{\mu_{j}}, \ldots, \mu_{n}\right)
$$




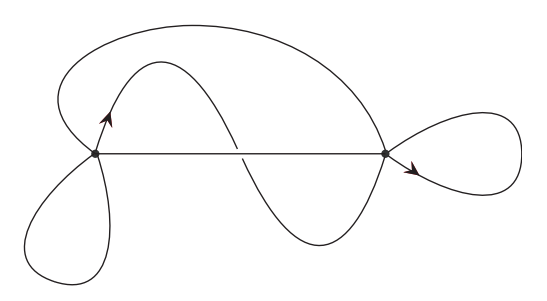

Figure 3.1. A cellular graph of type $(1,2)$.

$$
+\sum_{\alpha+\beta=\mu_{1}-2}\left[C_{g-1, n+1}\left(\alpha, \beta, \mu_{2}, \cdots, \mu_{n}\right)+\sum_{\substack{g_{1}+g_{2}=g \\ I \sqcup J=\{2, \ldots, n\}}} C_{g_{1},|I|+1}\left(\alpha, \mu_{I}\right) C_{g_{2},|J|+1}\left(\beta, \mu_{J}\right)\right],
$$

where $\mu_{I}=\left(\mu_{i}\right)_{i \in I}$ for an index set $I \subset[n],|I|$ denotes the cardinality of $I$, and the third sum in the formula is for all partitions of $g$ and set partitions of $\{2, \ldots, n\}$.

Proof. Let $\Gamma$ be an arrowed cellular graph counted by the left-hand side of $(3.2)$. Since all vertices of $\Gamma$ are labeled, let $\left\{p_{1}, \ldots, p_{n}\right\}$ denote the vertex set. We look at the half-edge incident to $p_{1}$ that carries an arrow.

Case 1. The arrowed half-edge extends to an edge $E$ that connects $p_{1}$ and $p_{j}$ for some $j>1$.

In this case, we shrink the edge and join the two vertices $p_{1}$ and $p_{j}$ together. By this process we create a new vertex of degree $\mu_{1}+\mu_{j}-2$. To make the counting bijective, we need to be able to go back from the shrunken graph to the original, provided that we know $\mu_{1}$ and $\mu_{j}$. Thus we place an arrow to the half-edge next to $E$ around $p_{1}$ with respect to the counter-clockwise cyclic order that comes from the orientation of the surface. In this process we have $\mu_{j}$ different arrowed graphs that produce the same result, because we must remove the arrow placed around the vertex $p_{j}$ in the original graph. This gives the right-hand side of the first line of (3.2). See Figure 3.2 .

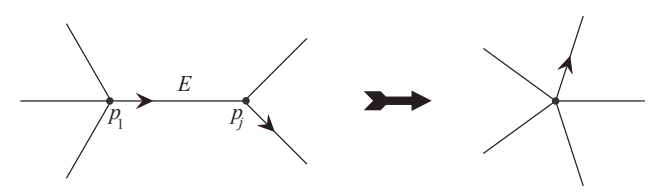

FiguRE 3.2. The process of shrinking the arrowed edge $E$ that connects vertices $p_{1}$ and $p_{j}, j>1$.

Case 2. The arrowed half-edge at $p_{1}$ is a loop $E$ that goes out and comes back to $p_{1}$.

The process we apply is again shrinking the loop $E$. The loop $E$ separates all other half-edges into two groups, one consisting of $\alpha$ of them placed on one side of the loop, and the other consisting of $\beta$ half-edges placed on the other side. It can happen that $\alpha=0$ or $\beta=0$. Shrinking a loop on a surface causes pinching. Instead of creating a pinched (i.e., singular) surface, we separate the double point into two new vertices of degrees $\alpha$ and $\beta$. Here again we need to remember the place of the loop $E$. Thus we place an arrow to the half-edge next to the loop in each group. See Figure 3.3 .

After the pinching and separating the double point, the original surface of genus $g$ with $n$ vertices $\left\{p_{1}, \ldots, p_{n}\right\}$ may change its topology. It may have genus $g-1$, or it splits into two 


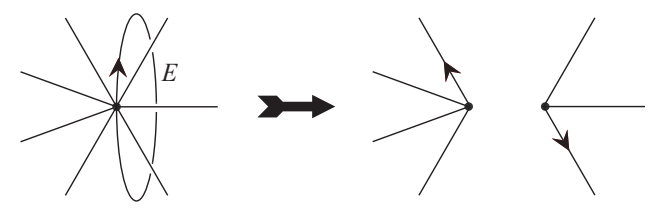

Figure 3.3. The process of shrinking the arrowed loop $E$ that is attached to $p_{1}$.

pieces of genus $g_{1}$ and $g_{2}$. The second line of (3.2) records all such cases. This completes the proof.

Remark 3.2. For $(g, n)=(0,1)$, the above formula reduces to

$$
C_{0,1}\left(\mu_{1}\right)=\sum_{\alpha+\beta=\mu_{1}-2} C_{0,1}(\alpha) C_{0,1}(\beta) .
$$

When $n=1$, the degree of the unique vertex $\mu_{1}$ is always even. By defining $C_{0,1}(0)=1$, we find that

$$
C_{0,1}(2 m)=C_{m}=\frac{1}{m+1}\left(\begin{array}{c}
2 m \\
m
\end{array}\right)
$$

is the $m$-th Catalan number. Only for $(g, n)=(0,1)$ we have this irregular case of $\mu_{1}=0$ happens, because a degree 0 single vertex is connected, and gives a cell-decomposition of $S^{2}$. We can imagine that a single vertex on $S^{2}$ has an infinite cyclic group as its automorphism, so that $C_{0,1}(0)=1$ is consistent with

$$
C_{0,1}\left(\mu_{1}\right)=\mu_{1} D_{0,1}\left(\mu_{1}\right) .
$$

All other cases, if one of the verteces has degree 0 , then the Catalan number $C_{g, n}$ is simply 0 because of the definition (3.1).

Let us introduce the generating function of the Catalan numbers by

$$
z=z(x)=\sum_{m=0}^{\infty} \frac{1}{x^{2 m+1}} .
$$

Then by the quadratic recursion (3.3) we find that the inverse function of $z(x)$ that vanishes at $x=\infty$ is given by

$$
x=z+\frac{1}{z} .
$$

This defines a Lagrangian immersion

$$
\Sigma=\mathbb{C} \ni z \longmapsto(x(z), y(z)) \in T^{*} \mathbb{C}, \quad\left\{\begin{array}{l}
x(z)=z+\frac{1}{z} \\
y(z)=-z
\end{array} .\right.
$$

The Lagrangian singularities are located at the points at which $d x=0$, i.e., $z= \pm 1$. Often it is more convenient to use the coordinate

$$
z=\frac{t+1}{t-1}
$$

The following theorem is established in [20].

Theorem 3.3 ([20]). The Laplace transform of the Catalan numbers of type $(g, n)$ defined as symmetric differential forms by

$$
W_{g, n}^{C}\left(t_{1}, \ldots, t_{n}\right)=(-1)^{n} \sum_{\left(\mu_{1}, \ldots, \mu_{n}\right) \in \mathbb{Z}_{+}^{n}} C_{g, n}\left(\mu_{1}, \ldots, \mu_{n}\right) e^{-\langle w, \mu\rangle} d w_{1} \cdots d w_{n}
$$


satisfies the Eynard-Orantin recursion with respect to the Lagrangian immersion (3.6) and

$$
W_{0,2}^{C}\left(t_{1}, t_{2}\right)=\frac{d t_{1} \cdot d t_{2}}{\left(t_{1}-t_{2}\right)^{2}}-\frac{d x_{1} \cdot d x_{2}}{\left(x_{1}-x_{2}\right)^{2}}
$$

Here the Laplace transform coordinate $w$ is related to the coordinate $t$ of the Lagrangian by

$$
e^{w_{i}}=x_{i}=z_{i}+\frac{1}{z_{i}}=\frac{t_{i}+1}{t_{i}-1}+\frac{t_{i}-1}{t_{i}+1}, \quad i=1,2, \ldots, n,
$$

and $\langle w, \mu\rangle=w_{1} \mu_{1}+\cdots+w_{n} \mu_{n}$.

In this case the Eynard-Orantin recursion formula is given by

$$
W_{g, n}^{C}\left(t_{1}, \ldots, t_{n}\right)=-\frac{1}{64} \frac{1}{2 \pi i} \int_{\gamma}\left(\frac{1}{t+t_{1}}+\frac{1}{t-t_{1}}\right) \frac{\left(t^{2}-1\right)^{3}}{t^{2}} \cdot \frac{1}{d t} \cdot d t_{1}
$$

$$
\begin{gathered}
\times\left[\sum_{j=2}^{n}\left(W_{0,2}^{C}\left(t, t_{j}\right) W_{g, n-1}^{C}\left(-t, t_{2}, \ldots, \widehat{t_{j}}, \ldots, t_{n}\right)+W_{0,2}^{D}\left(-t, t_{j}\right) W_{g, n-1}^{C}\left(t, t_{2}, \ldots, \widehat{t_{j}}, \ldots, t_{n}\right)\right)\right. \\
\left.+W_{g-1, n+1}^{C}\left(t,-t, t_{2}, \ldots, t_{n}\right)+\sum_{\substack{g_{1}+g_{2}=g \\
I \sqcup J=\{2,3, \ldots, n\}}}^{\text {stable }} W_{g_{1},|I|+1}^{C}\left(t, t_{I}\right) W_{g_{2},|J|+1}^{C}\left(-t, t_{J}\right)\right] .
\end{gathered}
$$

The last sum is restricted to the stable geometries. In other words, the partition should satisfies $2 g_{1}-1+|I|>0$ and $2 g_{2}-1+|J|$. The contour integral is taken with respect to $t$ on the curve defined by Figure 3.4

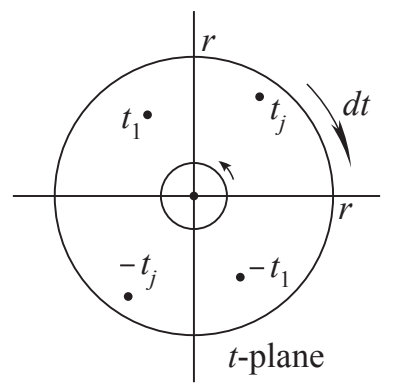

Figure 3.4. The integration contour $\gamma$. This contour encloses an annulus bounded by two concentric circles centered at the origin. The outer one has a large radius $r>$ $\max _{j \in N}\left|t_{j}\right|$ and the negative orientation, and the inner one has an infinitesimally small radius with the positive orientation.

Remark 3.4. The recursion formula (3.10) is a genuine induction formula with respect to $2 g-2+n$. Thus from the given $W_{0,1}^{C}$ and $W_{0,2}^{C}$, we can calculate all $W_{g, n}^{C}$ one by one. This is a big difference between (3.10) and (3.2). The latter relation contains the terms with $C_{g, n}$ in the right-hand side as well.

\section{The partition function for the generalized Catalan numbers and the SCHRÖDINGER EQUATION}

Let us now define

$$
F_{g, n}^{C}\left(t_{1}, \ldots, t_{n}\right)=\sum_{\left(\mu_{1}, \ldots, \mu_{n}\right) \in \mathbb{Z}_{+}^{n}} D_{g, n}\left(\mu_{1}, \ldots, \mu_{n}\right) e^{-\langle w, \mu\rangle}
$$


for $2 g-2+n>0$. Then from (3.1) we have

$$
W_{g, n}^{C}\left(t_{1}, \ldots, t_{n}\right)=d_{1} \cdots d_{n} F_{g, n}^{C}\left(t_{1}, \ldots, t_{n}\right) .
$$

Therefore, we have a natural primitive function of $W_{g, n}\left(t_{1}, \ldots, t_{n}\right)$ for every $(g, n)$. We note that

$$
t=-1 \Longrightarrow z=0 \Longrightarrow x=\infty \text {. }
$$

Therefore,

$$
\left.F_{g, n}^{C}\left(t_{1}, \ldots, t_{n}\right)\right|_{t_{i}=-1}=0
$$

for every $i=1,2, \ldots, n$. The following recursion formula of [61] is the key for our investigation.

Theorem 4.1. The Laplace transform $F_{g, n}^{C}\left(t_{[n]}\right)$ satisfies the following differential recursion equation for every $(g, n)$ subject to $2 g-2+n>0$.

$$
\begin{aligned}
& \frac{\partial}{\partial t_{1}} F_{g, n}^{C}\left(t_{[n]}\right) \\
& =-\frac{1}{16} \sum_{j=2}^{n}\left[\frac{t_{j}}{t_{1}^{2}-t_{j}^{2}}\left(\frac{\left(t_{1}^{2}-1\right)^{3}}{t_{1}^{2}} \frac{\partial}{\partial t_{1}} F_{g, n-1}^{C}\left(t_{[\hat{j}]}\right)-\frac{\left(t_{j}^{2}-1\right)^{3}}{t_{j}^{2}} \frac{\partial}{\partial t_{j}} F_{g, n-1}^{C}\left(t_{[\hat{i}]}\right)\right)\right] \\
& -\frac{1}{16} \sum_{j=2}^{n} \frac{\left(t_{1}^{2}-1\right)^{2}}{t_{1}^{2}} \frac{\partial}{\partial t_{1}} F_{g, n-1}^{C}\left(t_{[\hat{j}]}\right) \\
& -\left.\frac{1}{32} \frac{\left(t_{1}^{2}-1\right)^{3}}{t_{1}^{2}}\left[\frac{\partial^{2}}{\partial u_{1} \partial u_{2}} F_{g-1, n+1}^{C}\left(u_{1}, u_{2}, t_{2}, t_{3}, \ldots, t_{n}\right)\right]\right|_{u_{1}=u_{2}=t_{1}} \\
& -\frac{1}{32} \frac{\left(t_{1}^{2}-1\right)^{3}}{t_{1}^{2}} \sum_{\substack{g_{1}+g_{2}=g \\
I \sqcup J=\{2,3, \ldots, n\}}}^{\text {stable }} \frac{\partial}{\partial t_{1}} F_{g_{1},|I|+1}^{C}\left(t_{1}, t_{I}\right) \frac{\partial}{\partial t_{1}} F_{g_{2},|J|+1}^{C}\left(t_{1}, t_{J}\right) .
\end{aligned}
$$

Here we use the index convention $[n]=\{1,2, \ldots, n\}$ and $[\hat{j}]=\{1,2, \ldots, \hat{j}, \ldots, n\}$.

Remark 4.2. We note that the above formula is identical to [58, Theorem 5.1], even though $F_{g, n}$ is a different function. There we considered the Laplace transform of the number of lattice points in $\mathcal{M}_{g, n}$, and hence $F_{0,1}=F_{0,2}=0$.

Remark 4.3. Because of $(4.2)$, the recursion 4.3 uniquely determines each $F_{g, n}^{C}$ by integrating from -1 to $t_{1}$. With the same reason, $F_{g, n}^{C}$ is uniquely determined by $W_{g, n}^{C}$. Since we know exactly where $F_{g, n}^{C}$ vanishes, there is no discrepancy of the constants of integration in 2.12 .

Let us now consider the principal specialization of the partition function for the Catalan numbers

$$
Z^{C}(t, \hbar)=\exp \left(\sum_{g=0}^{\infty} \sum_{n=1}^{\infty} \frac{1}{n !} \hbar^{2 g-2+n} F_{g, n}^{C}(t, t, \ldots, t)\right) .
$$

Since unstable terms $F_{0,1}^{C}(t)$ and $F_{0,2}^{C}(t, t)$ are included in the above formula, we need to calculate them first. 
Proposition 4.4. In terms of the z-variable, we have

$$
\begin{aligned}
F_{0,1}^{C}(t) & =-\frac{1}{2} z^{2}+\log z, \\
F_{0,2}^{C}\left(t_{1}, t_{2}\right) & =-\log \left(1-z_{1} z_{2}\right) .
\end{aligned}
$$

Proof. Due to the irregularity of $\mu=0$ for $D_{0,1}(\mu)$, we need to modify the definitions 3.8 and 4.1 for $(g, n)=(0,1)$. It is natural to define

$$
W_{0,1}(t)=-\sum_{m=0}^{\infty} C_{0,1}(2 m) \frac{d x}{x^{2 m+1}}=-z d x=\left(-z+\frac{1}{z}\right) d z
$$

because of the consistency with $(2.7)$. Since $W_{0,1}^{C}=d F_{0,1}^{C}$, we have

$$
\begin{aligned}
F_{0,1}(t) & =-\frac{1}{2} z^{2}+\log z+\text { const } \\
& =\sum_{m=0}^{\infty} D_{0,1}(2 m)\left(\frac{1}{x^{2 m}}-\delta_{m, 0}\right),
\end{aligned}
$$

where the $m=0$ term is adjusted so that we do not have the infinity term $D_{0,1}(0)$ in $F_{0,1}^{C}$. Using the expression

$$
D_{0,1}(2 m)=\frac{1}{2 m(m+1)}\left(\begin{array}{c}
2 m \\
m
\end{array}\right)
$$

we have

$$
\lim _{m \rightarrow 0} D_{0,1}(2 m)\left(\frac{1}{x^{2 m}}-\delta_{m, 0}\right)=-\log x .
$$

Since $x=z+\frac{1}{z}$, by taking the limit $z \rightarrow 0$ we conclude that the constant term in $F_{0,1}^{C}(t)$ is 0 , which establishes 4.5 ).

The computation of $F_{0,1}^{C}\left(t_{1}, t_{2}\right)$ is performed in [20, Proposition 4.1], where the idea is to use the Euler differential operator $x_{1} \frac{d}{d x_{1}}+x_{2} \frac{d}{d x_{2}}$. By definition $F_{0,1}^{C}\left(t_{1}, t_{2}\right)$ does not have any constant term as $x \rightarrow \infty$, therefore there is no constant correction in (4.6), either.

Theorem 4.5. The principal specialization of the partition function satisfies the following Schrödinger equation.

$$
\left(\hbar^{2} \frac{d^{2}}{d x^{2}}+\hbar x \frac{d}{d x}+1\right) Z^{C}(t, \hbar)=0
$$

where $t$ is considered as a function in $x$ by

$$
t=t(x)=\frac{z(x)+1}{z(x)-1}
$$

and (3.4).

The rest of this section is devoted to proving the above theorem. Let us define for $m \geq 0$

$$
S_{m}=\sum_{2 g-2+n=m-1} \frac{1}{n !} F_{g, n}^{C}(t, \ldots, t),
$$

and put

$$
F=\sum_{m=0}^{\infty} \hbar^{m-1} S_{m}
$$


Then the Schrödinger equation (4.8) becomes

$$
\hbar^{2}\left(\frac{d^{2} F}{d x^{2}}+\left(\frac{d F}{d x}\right)^{2}\right)+\hbar x \frac{d F}{d x}+1=0
$$

which is equivalent to

$$
\sum_{m=0}^{\infty} S_{m}^{\prime \prime} \hbar^{m+1}+\left(\sum_{m=0}^{\infty} S_{m}^{\prime} \hbar^{m}\right)^{2}+x \sum_{m=0}^{\infty} S_{m}^{\prime} \hbar^{m}+1=0
$$

where $^{\prime}=\frac{d}{d x}$. Since $S_{0}=F_{0,1}^{C}$ and

$$
W_{0,1}^{C}=d F_{0,1}^{C}=y d x=-z d x,
$$

we obtain

$$
S_{0}^{\prime}=\frac{d}{d x} F_{0,1}^{C}=-z=-\frac{t+1}{t-1} .
$$

Using the Lagrangian immersion (3.5), we see that the constant terms of 4.10 then become

$$
(-z)^{2}+x(-z)+1=z^{2}-x z+1=0 .
$$

Collecting the $\hbar^{m+1}$-contributions in 4.10 for $m \geq 0$, we obtain

$$
\begin{aligned}
-x \frac{d}{d x} S_{m+1} & =\frac{d^{2}}{d x^{2}} S_{m}+\sum_{a+b=m+1} \frac{d S_{a}}{d x} \frac{d S_{b}}{d x} \\
& =\frac{d^{2}}{d x^{2}} S_{m}+2 S_{0}^{\prime} S_{m+1}^{\prime}+2 S_{1}^{\prime} S_{m}^{\prime}+\sum_{\substack{a+b=m+1 \\
a, b \geq 2}} \frac{d S_{a}}{d x} \frac{d S_{b}}{d x} .
\end{aligned}
$$

Therefore,

$$
-\left(2 S_{0}^{\prime}+x\right) \frac{d}{d x} S_{m+1}=\frac{d^{2}}{d x^{2}} S_{m}+2 S_{1}^{\prime} \frac{d}{d x} S_{m}+\sum_{\substack{a+b=m+1 \\ a, b \geq 2}} \frac{d S_{a}}{d x} \frac{d S_{b}}{d x} .
$$

To use the closed formulas 4.5) and 4.6 for $S_{0}$ and $S_{1}$ here, we need to switch from the $x$-coordinate to the $t$-coordinate. Using the change of variable formulas (3.5) and (3.7), we have

$$
d x=d z\left(1-\frac{1}{z^{2}}\right)=-\frac{8 t}{\left(t^{2}-1\right)^{2}} d t
$$

hence

Therefore,

$$
\begin{aligned}
\frac{d}{d x} & =\frac{z^{2}}{z^{2}-1} \frac{d}{d z}=-\frac{\left(t^{2}-1\right)^{2}}{8 t} \frac{d}{d t}, \\
\frac{d^{2}}{d x^{2}} & =\frac{\left(t^{2}-1\right)^{4}}{64 t^{2}} \frac{d^{2}}{d t^{2}}+\frac{\left(t^{2}-1\right)^{2}}{8 t}\left(\frac{d}{d t} \frac{\left(t^{2}-1\right)^{2}}{8 t}\right) \frac{d}{d t} \\
& =\frac{\left(t^{2}-1\right)^{4}}{64 t^{2}} \frac{d^{2}}{d t^{2}}+\frac{\left(t^{2}-1\right)^{3}}{64 t^{3}}\left(3 t^{2}+1\right) \frac{d}{d t} .
\end{aligned}
$$

$$
-\left(2 S_{0}^{\prime}+x\right) \frac{d}{d x}=-\frac{\left(t^{2}-1\right)^{2}}{8 t}\left(z-\frac{1}{z}\right) \frac{d}{d t}=-\frac{\left(t^{2}-1\right)^{2}}{8 t} \frac{4 t}{t^{2}-1} \frac{d}{d t}=\frac{1}{2}\left(t^{2}-1\right) \frac{d}{d t},
$$




$$
S_{1}^{\prime}=\frac{1}{2} \frac{d}{d x} F_{0,2}^{C}(z, z)=\frac{1}{2} \frac{z^{2}}{z^{2}-1} \frac{d}{d z}\left(-\log \left(1-z^{2}\right)\right)=-\frac{\left(t^{2}-1\right)(t+1)^{2}}{16 t^{2}} .
$$

Substituting 4.11), 4.14 and 4.15) in 4.13), we obtain

Proposition 4.6. For $m \geq 0$, the Schrödinger equation $(4.8)$ is equivalent to a recursion formula

$$
\frac{d}{d t} S_{m+1}=-\frac{\left(t^{2}-1\right)^{3}}{32 t^{2}}\left[\frac{d^{2}}{d t^{2}} S_{m}+\sum_{\substack{a+b=m+1 \\ a, b \geq 2}} \frac{d S_{a}}{d t} \frac{d S_{b}}{d t}\right]-\frac{\left(t^{2}-1\right)^{2}}{16 t^{3}}\left(2 t^{2}+t+1\right) \frac{d}{d t} S_{m}
$$

Remark 4.7. The recursion equation $(4.16)$ is a direct consequence of the principal specialization applied to 4.3 .

The proof of 4.16 is given in Appendix A

\section{Single Hurwitz Numbers}

The A-model problem that we are interested in this section is the automorphism-weighted count $H_{g, n}\left(\mu_{1}, \ldots, \mu_{n}\right)$ of the number of the topological types of meromorphic functions $f: C \longrightarrow \mathbb{P}^{1}$ of a nonsingular complete irreducible algebraic curve $C$ of genus $g$ that has $n$ labeled poles of orders $\left(\mu_{1}, \ldots, \mu_{n}\right)$ such that all other critical points of $f$ than these poles are unlabeled simple ramification points. Let $r$ denote the number of such simple ramification points. Then the Riemann-Hurwitz formula gives

$$
r=2 g-2+n+\sum_{i=1}^{n} \mu_{i} .
$$

A remarkable formula due to Ekedahl, Lando, Shapiro and Vainshtein [22, 37, 68] relates Hurwitz numbers and Gromov-Witten invariants. For genus $g \geq 0$ and the number $n \geq 1$ of the poles subject to the stability condition $2 g-2+n>0$, the ELSV formula states that

$$
\begin{aligned}
& H_{g, n}\left(\mu_{1}, \ldots, \mu_{n}\right)=\prod_{i=1}^{n} \frac{\mu_{i}^{\mu_{i}}}{\mu_{i} !} \int_{\overline{\mathcal{M}}_{g, n}} \frac{\Lambda_{g}^{\vee}(1)}{\prod_{i=1}^{n}\left(1-\mu_{i} \psi_{i}\right)} \\
&=\sum_{j=0}^{g}(-1)^{j} \sum_{k_{1}, \ldots, k_{n} \geq 0}\left\langle\tau_{k_{1}} \cdots \tau_{k_{n}} c_{j}(\mathbb{E})\right\rangle_{g, n} \prod_{i=1}^{n} \frac{\mu_{i}^{\mu_{i}+k_{i}}}{\mu_{i} !},
\end{aligned}
$$

where $\overline{\mathcal{M}}_{g, n}$ is the Deligne-Mumford moduli stack of stable algebraic curves of genus $g$ with $n$ distinct smooth marked points, $\Lambda_{g}^{\vee}(1)=1-c_{1}(\mathbb{E})+\cdots+(-1)^{g} c_{g}(\mathbb{E})$ is the alternating sum of the Chern classes of the Hodge bundle $\mathbb{E}$ on $\overline{\mathcal{M}}_{g, n}, \psi_{i}$ is the $i$-th tautological cotangent class, and

$$
\left\langle\tau_{k_{1}} \cdots \tau_{k_{n}} c_{j}(\mathbb{E})\right\rangle_{g, n}=\int_{\overline{\mathcal{M}}_{g, n}} \psi_{1}^{k_{1}} \cdots \psi_{n}^{k_{n}} c_{j}(\mathbb{E})
$$

is the linear Hodge integral, which is 0 unless $k_{1}+\cdots+k_{n}+j=3 g-3+n$. Although the Deligne-Mumford stack $\overline{\mathcal{M}}_{g, n}$ is not defined for $2-2 g-n<0$, single Hurwitz numbers are well defined for unstable geometries $(g, n)=(0,1)$ and $(0,2)$, and their values are

$$
H_{0}((d))=\frac{d^{d-3}}{(d-1) !}=\frac{d^{d-2}}{d !} \quad \text { and } \quad H_{0}\left(\left(\mu_{1}, \mu_{2}\right)\right)=\frac{1}{\mu_{1}+\mu_{2}} \cdot \frac{\mu_{1}^{\mu_{1}}}{\mu_{1} !} \cdot \frac{\mu_{2}^{\mu_{2}}}{\mu_{2} !} .
$$


The ELSV formula remains valid for unstable cases if we define

$$
\begin{aligned}
& \int_{\overline{\mathcal{M}}_{0,1}} \frac{\Lambda_{0}^{\vee}(1)}{1-d \psi}=\frac{1}{d^{2}}, \\
& \int_{\overline{\mathcal{M}}_{0,2}} \frac{\Lambda_{0}^{\vee}(1)}{\left(1-\mu_{1} \psi_{1}\right)\left(1-\mu_{2} \psi_{2}\right)}=\frac{1}{\mu_{1}+\mu_{2}} .
\end{aligned}
$$

The ELSV formula predicts that the single Hurwitz numbers exhibit the polynomial behavior in terms of their Laplace transform. Following [26], and modifying our choice of the coordinates slightly, we define

$$
\begin{gathered}
F_{g, n}^{H}\left(t_{1}, \ldots, t_{n}\right)=\sum_{\mu \in \mathbb{Z}_{+}^{n}} H_{g, n}\left(\mu_{1}, \ldots, \mu_{n}\right) e^{-\left(\mu_{1} w_{1}+\cdots+\mu_{n} w_{n}\right)} \\
=\sum_{\mu \in \mathbb{Z}_{+}^{n}} \sum_{k_{1}+\cdots+k_{n} \leq 3 g-3+n}\left\langle\tau_{k_{1}} \cdots \tau_{k_{n}} \Lambda_{g}^{\vee}(1)\right\rangle_{g, n}\left(\prod_{i=1}^{n} \frac{\mu_{i}^{\mu_{i}+k_{i}}}{\mu_{i} !}\right) e^{-\left(\mu_{1} w_{1}+\cdots+\mu_{n} w_{n}\right)} \\
=\sum_{k_{1}+\cdots+k_{n} \leq 3 g-3+n}\left\langle\tau_{k_{1}} \cdots \tau_{k_{n}} \Lambda_{g}^{\vee}(1)\right\rangle_{g, n} \prod_{i=1}^{n} \hat{\xi}_{k_{i}}\left(t_{i}\right),
\end{gathered}
$$

where the $\hat{\xi}$-functions are given by

$$
\hat{\xi}_{k}(t)=\sum_{\mu=1}^{\infty} \frac{\mu^{\mu+k}}{\mu !} e^{-\mu w}=\sum_{\mu=1}^{\infty} \frac{\mu^{\mu+k}}{\mu !} x^{\mu}
$$

for $k \geq 0$, and $x=e^{-w}$. Although $\hat{\xi}_{k}$ are complicated functions in $x$, their behavior is simple in terms of $\hat{\xi}_{0}$. So we introduce

$$
t=1+\sum_{\mu=1}^{\infty} \frac{\mu^{\mu}}{\mu !} x^{\mu} \quad \text { and } \quad z=\sum_{\mu=1}^{\infty} \frac{\mu^{\mu-1}}{\mu !} x^{\mu} .
$$

Then by the Lagrange inversion formula, we have

$$
x=z e^{-z} \quad \text { and } \quad z=\frac{t-1}{t},
$$

and moreover, each $\hat{\xi}_{k}(t)$ is a polynomial of degree $2 k+1$ in $t$ for every $k \geq 0$, recursively defined by

$$
\hat{\xi}_{k+1}(t)=t^{2}(t-1) \frac{d}{d t} \hat{\xi}_{k}(t)
$$

This is because

$$
-\frac{d}{d w}=x \frac{d}{d x}=t^{2}(t-1) \frac{d}{d t} .
$$

Therefore, if $2 g-2+n>0$, then $F_{g, n}^{H}\left(t_{1}, \ldots, t_{n}\right)$ is a symmetric polynomial of degree $6 g-6+3 n$, and satisfies

$$
\left.F_{g, n}^{H}\left(t_{1}, \ldots, t_{n}\right)\right|_{t_{j}=1}=0
$$

for every $j=1,2 \ldots, n$.

The computation of [20] adjusted to our current convention of this paper,

$$
F_{0,1}^{H}(t)=\frac{1}{2}\left(1-\frac{1}{t^{2}}\right)=z-\frac{1}{2} z^{2}
$$


and

$$
F_{0,2}^{H}\left(t_{1}, t_{2}\right)=\log \left(\frac{z_{1}-z_{2}}{x_{1}-x_{2}}\right)-\left(z_{1}+z_{2}\right),
$$

determines the Lagrangian immersion

$$
\iota: \Sigma=\mathbb{C}^{*} \longrightarrow T^{*} \mathbb{C}^{*}
$$

by

$$
\left\{\begin{array}{l}
x=z e^{-z} \\
y=z
\end{array}\right.
$$

and

$$
W_{0,2}^{H}\left(t_{1}, t_{2}\right)=d_{1} \otimes d_{2} F_{0,2}^{H}\left(t_{1}, t_{2}\right)=\frac{d t_{1} \cdot d t_{2}}{\left(t_{1}-t_{2}\right)^{2}}-\frac{d x_{1} \cdot d x_{2}}{\left(x_{1}-x_{2}\right)^{2}} .
$$

Here the tautological 1-form on $T^{*} \mathbb{C}^{*}$ is chosen to be

$$
\eta=y \frac{d x}{x} \text {. }
$$

It is consistent with

$$
W_{0,1}^{H}(t)=d F_{0,1}^{H}(t)=z \frac{d x}{x}=\iota^{*} \eta .
$$

We also note that

$$
\left.F_{0,1}^{H}(t)\right|_{t=1}=0
$$

and

$$
\left.F_{0,2}^{H}\left(t_{1}, t_{2}\right)\right|_{t_{j}=1}=0, \quad j=1 \text { or } 2 .
$$

The latter equality holds because $t_{2}=1 \Longrightarrow z_{2}=0 \Longrightarrow x_{2}=0$, and hence from $(5.15)$ we have

Since

$$
F_{0,2}^{H}\left(t_{1}, 1\right)=\log \frac{z_{1}}{x_{1}}-z_{1}=0 .
$$

$$
\left.\frac{x_{1}-x_{2}}{z_{1}-z_{2}}\right|_{z_{1}=z_{2}=z}=(1-z) e^{-z}
$$

the diagonal value of $F_{0,2}^{H}$ is calculated as

$$
F_{0,2}^{H}(t, t)=\log \left(\frac{e^{z}}{-z}\right)-2 z=-z-\log (1-z)=\frac{1-t}{t}+\log t .
$$

The single Hurwitz numbers $H_{g, n}(\vec{\mu})$ satisfy the cut-and-join equation [36, 77 ]

$$
\begin{gathered}
\left(2 g-2+n+\sum_{i=1}^{n} \mu_{i}\right) H_{g, n}\left(\mu_{1}, \ldots, \mu_{n}\right)=\frac{1}{2} \sum_{i \neq j}\left(\mu_{i}+\mu_{j}\right) H_{g, n-1}\left(\mu_{i}+\mu_{j}, \mu_{[\hat{i}, \hat{j}]}\right) \\
+\frac{1}{2} \sum_{i=1}^{n} \sum_{\alpha+\beta=\mu_{i}} \alpha \beta\left[H_{g-1, n+1}\left(\alpha, \beta, \mu_{[\hat{i}]}\right)+\sum_{\substack{g_{1}+g_{2}=g \\
I \sqcup J=[\hat{i}]}} H_{g_{1},|I|+1}\left(\alpha, \mu_{I}\right) H_{g_{2},|J|+1}\left(\beta, \mu_{J}\right)\right],
\end{gathered}
$$

where we use the convention for indices as in Section 3. The Laplace transform of (5.21) is the polynomial recursion of [60] and takes the form 


$$
\begin{aligned}
& \left(2 g-2+n+\sum_{i=1}^{n} t_{i}\left(t_{i}-1\right) \frac{\partial}{\partial t_{i}}\right) F_{g, n}^{H}\left(t_{1}, \ldots, t_{n}\right) \\
& =\frac{1}{2} \sum_{i \neq j} \frac{t_{i} t_{j}}{t_{i}-t_{j}}\left(t_{i}^{2}\left(t_{i}-1\right)^{2} \frac{\partial}{\partial t_{i}} F_{g, n-1}^{H}\left(t_{[\hat{j}]}\right)-t_{j}^{2}\left(t_{j}-1\right)^{2} \frac{\partial}{\partial t_{j}} F_{g, n-1}^{H}\left(t_{[\hat{i}]}\right)\right) \\
& \quad-\sum_{i \neq j} t_{i}^{3}\left(t_{i}-1\right) \frac{\partial}{\partial t_{i}} F_{g, n-1}^{H}\left(t_{[\hat{j}]}\right) \\
& +\left.\frac{1}{2} \sum_{i=1}^{n}\left(t_{i}^{2}\left(t_{i}-1\right)\right)^{2} \frac{\partial^{2}}{\partial u_{1} \partial u_{2}} F_{g-1, n+1}^{H}\left(u_{1}, u_{2}, t_{[\hat{i}]}\right)\right|_{u_{1}=u_{2}=t_{i}} \\
& +\frac{1}{2} \sum_{i=1}^{n}\left(t_{i}^{2}\left(t_{i}-1\right)\right)^{2} \sum_{\substack{g_{1}+g_{2}=g \\
I \sqcup J=[\hat{i}]}}^{\text {stable }} \frac{\partial}{\partial t_{i}} F_{g_{1},|I|+1}^{H}\left(t_{i}, t_{I}\right) \cdot \frac{\partial}{\partial t_{i}} F_{g_{1},|J|+1}^{H}\left(t_{i}, t_{J}\right) .
\end{aligned}
$$

It is proved in 26] that the $n$-fold exterior differentiation of the above formula is exactly the Eynard-Orantin recursion, as predicted by Bouchard and Mariño [9]. Thus we obtain a natural integration of the Eynard-Orantin recursion by taking the Laplace transform of the A-model quantity again, which is the single Hurwitz umber $H_{g, n}(\vec{\mu})$.

Theorem 5.1. Let us define

$$
S_{m}^{H}(t)=\sum_{2 g-2+n=m-1} \frac{1}{n !} F_{g, n}^{H}(t, \ldots, t) .
$$

Then $S_{m}^{H}$ 's satisfy the following second order differential equations:

$$
\left(e^{m w} \frac{d}{d w} e^{-m w}\right) S_{m+1}^{H}=-\frac{1}{2}\left[\frac{d^{2}}{d w^{2}} S_{m}^{H}+\sum_{a+b=m+1} \frac{d S_{a}^{H}}{d w} \cdot \frac{d S_{b}^{H}}{d w}+\frac{d}{d w} S_{m}^{H}\right] .
$$

Here the $w$-dependence of $t$ is given by $x=e^{-w}$ and (5.9). We also note that $S_{m}^{H}(t)$ is a polynomial of degree $3 m-3$ for every $m \geq 2$, and for all values of $m$ we have

$$
\left.S_{m}^{H}(t)\right|_{t=1}=0 \text {. }
$$

The proof is similar to the case of the Catalan numbers (Section 4 and Appendix A). First we compute the principal specialization of the differential equation (5.22). We then assemble them according to (5.23). By adjusting the unstable geometry terms $(g, n)=(0,1)$ and $(0,2)$, we obtain (5.24). However, due to the difference between the cut-and-join equation and the edge-shrinking operation of Section 3 , the resulting equation becomes quite different.

Choose $m \geq 2$ and $(g, n)$ so that $2 g-2+n=m$. Then the principal specialization of the left-hand side of $(5.22)$ is

$$
\left(m+t(t-1) \frac{d}{d t}\right) F_{g, n}^{H}(t, \ldots, t) .
$$

The first line of the right-hand side of $(5.22)$ gives

$$
\begin{aligned}
\left.\frac{1}{2} t^{2} \sum_{i \neq j} \frac{\partial}{\partial t_{i}}\left(t_{i}^{2}\left(t_{i}-1\right)^{2} \frac{\partial}{\partial t_{i}} F_{g, n-1}^{H}\left(t_{i}, t, \ldots, t\right)\right)\right|_{t_{i}=t} \\
=\frac{n(n-1)}{2} t^{2} \frac{d}{d t}\left(t^{2}(t-1)^{2}\right) \cdot \frac{1}{n-1} \frac{d}{d t} F_{g, n-1}^{H}(t, \ldots, t)
\end{aligned}
$$




$$
\begin{aligned}
& +\left.\frac{n(n-1)}{2} t^{4}(t-1)^{2} \frac{\partial^{2}}{\partial u^{2}} F_{g, n-1}^{H}(u, t, \ldots, t)\right|_{u=t} \\
& =\frac{1}{2} n ! t^{2} \frac{d}{d t}\left(t^{2}(t-1)^{2}\right) \cdot \frac{1}{(n-1) !} \frac{d}{d t} F_{g, n-1}^{H}(t, \ldots, t) \\
& \quad+\left.\frac{1}{2} n !(n-1) t^{4}(t-1)^{2} \frac{1}{(n-1) !} \frac{\partial^{2}}{\partial u^{2}} F_{g, n-1}^{H}(u, t, \ldots, t)\right|_{u=t} .
\end{aligned}
$$

The second line of the right-hand side of $(5.22)$ becomes

$$
-n ! t^{3}(t-1) \frac{1}{(n-1) !} \frac{d}{d t} F_{g, n-1}^{H}(t, \ldots, t) .
$$

The third line simply produces

$$
\left.\frac{n !}{2}\left(t^{2}(t-1)\right)^{2} \frac{1}{(n+1) !}(n+1) n \frac{\partial^{2}}{\partial u_{1} \partial u_{2}} F_{g-1, n+1}^{H}\left(u_{1}, u_{2}, t \ldots, t\right)\right|_{u_{1}=u_{2}=t} .
$$

Finally, since the set partition becomes the partition of numbers because all variables are set to be equal, the fourth line of the right-hand side of 5.22 gives

$$
\frac{n !}{2}\left(t^{2}(t-1)\right)^{2} \sum_{\substack{g_{1}+g_{2}=g \\ n_{1}+n_{2}=n-1}}^{\text {stable }} \frac{1}{\left(n_{1}+1\right) !} \frac{d}{d t} F_{g_{1}, n_{1}+1}^{H}(t, \ldots, t) \cdot \frac{1}{\left(n_{2}+1\right) !} \frac{d}{d t} F_{g_{1}, n_{2}+1}^{H}(t, \ldots, t) .
$$

We now apply the operation

$$
\sum_{2 g-2+n=m} \frac{1}{n !}
$$

to the above terms. The left-hand side becomes

$$
\left(m+t(t-1) \frac{d}{d t}\right) S_{m+1}^{H}
$$

The right-hand side terms are re-assembled into the sum of $\left(g^{\prime}, n^{\prime}\right)$ subject to $2 g^{\prime}-2+n^{\prime}=$ $m-1$, following the topological structure of the recursion (5.22). Noticing that unstable geometers are contained only in $S_{0}^{H}$ and $S_{1}^{H}$, we obtain

$$
\begin{aligned}
& \left(m+t(t-1) \frac{d}{d t}\right) S_{m+1}^{H}(t) \\
= & \frac{1}{2}\left(t^{2} \frac{d}{d t}\left(t^{2}(t-1)^{2}\right) \cdot \frac{d}{d t} S_{m}^{H}(t)+\left(t^{2}(t-1)\right)^{2} \frac{d^{2}}{d t^{2}} S_{m}^{H}(t)-2 t^{3}(t-1) \frac{d}{d t} S_{m}^{H}(t)\right) \\
& +\frac{1}{2}\left(t^{2}(t-1)^{2}\right)^{2} \sum_{\substack{a+b=m+1 \\
a, b \geq 2}} \frac{d}{d t} S_{a}^{H}(t) \cdot \frac{d}{d t} S_{b}^{H}(t) \\
= & \frac{1}{2}\left(t^{2}(t-1)\right)^{2}\left(\frac{d^{2}}{d t^{2}} S_{m}^{H}(t)+\sum_{\substack{a+b=m+1 \\
a, b \geq 2}} \frac{d}{d t} S_{a}^{H}(t) \cdot \frac{d}{d t} S_{b}^{H}(t)\right)+2 t^{3}(t-1)^{2} \frac{d}{d t} S_{m}^{H}(t) .
\end{aligned}
$$

Proposition 5.2. The functions $S_{m}(t)$ are recursively determined by

$$
S_{0}^{H}(t)=\frac{1}{2}\left(1-\frac{1}{t^{2}}\right)
$$




$$
S_{1}^{H}(t)=\frac{1}{2}\left(\frac{1-t}{t}+\log t\right)
$$

and

$$
\begin{array}{r}
S_{m+1}^{H}(t)=\left(\frac{t-1}{t}\right)^{-m} \int_{1}^{t}\left[\frac { 1 } { 2 } t ^ { 3 - m } ( t - 1 ) ^ { m + 1 } \left(\frac{d^{2}}{d t^{2}} S_{m}^{H}(t)\right.\right. \\
+\sum_{\substack{a+b=m+1 \\
a, b \geq 2}} \frac{d}{d t} S_{a}^{H}(t) \cdot \frac{d}{d t} S_{b}^{H}(t) \\
\left.+2 t^{2-m}(t-1)^{m+1} \frac{d}{d t} S_{m}^{H}(t)\right] d t .
\end{array}
$$

Proof. As a differential operator,

$$
m+t(t-1) \frac{d}{d t}=t(t-1)\left(\frac{t-1}{t}\right)^{-m} \frac{d}{d t}\left(\frac{t-1}{t}\right)^{m} .
$$

Therefore, (5.26) is equivalent to

$$
\begin{aligned}
& {\left[\left(\frac{t-1}{t}\right)^{-m} \frac{d}{d t}\left(\frac{t-1}{t}\right)^{m}\right] S_{m+1}^{H}(t)} \\
& \quad=\frac{1}{2} t^{3}(t-1)\left(\frac{d^{2}}{d t^{2}} S_{m}^{H}(t)+\sum_{\substack{a+b=m+1 \\
a, b \geq 2}} \frac{d}{d t} S_{a}^{H}(t) \cdot \frac{d}{d t} S_{b}^{H}(t)\right)+2 t^{2}(t-1) \frac{d}{d t} S_{m}^{H}(t) .
\end{aligned}
$$

On the right-hand side only $S_{k}^{H}(t)$ with $k \leq m$ appear. Using the fact of the zero (5.25), we obtain $(5.29)$.

On the third line of 5.26 the terms with $S_{0}^{H}$ and $S_{1}^{H}$ are not included. More precisely, these omitted terms are

$$
t(t-1)^{2} \frac{d}{d t} S_{m+1}^{H}(t)+\frac{1}{2} t^{2}(t-1)^{3} \frac{d}{d t} S_{m}^{H}(t) .
$$

When we add these terms to 5.26), and adjust the second order differentiation as

$$
\left(t^{2}(t-1) \frac{d}{d t}\right)^{2}=\left(t^{2}(t-1)\right)^{2} \frac{d^{2}}{d t^{2}}+t^{3}(t-1)(3 t-2) \frac{d}{d t},
$$

we finally obtain

$\left(m+t^{2}(t-1) \frac{d}{d t}\right) S_{m+1}^{H}(t)$

$$
=\frac{1}{2}\left(\left(t^{2}(t-1) \frac{d}{d t}\right)^{2} S_{m}^{H}(t)+\left(t^{2}(t-1)\right)^{2} \sum_{a+b=m+1} \frac{d S_{a}^{H}(t)}{d t} \cdot \frac{d S_{b}^{H}(t)}{d t}-t^{2}(t-1) \frac{d}{d t} S_{m}^{H}(t)\right) .
$$

Then (5.24) follows from (5.30) and (5.12). This completes the proof Theorem 5.1. 
Theorem 5.3. Let us define the partition function for the single Hurwitz numbers in a similar way:

$$
Z^{H}(t, \hbar)=\exp \left(\sum_{m=0}^{\infty} S_{m}^{H} \hbar^{m-1}\right)=\exp \left(\sum_{g=0}^{\infty} \sum_{n=1}^{\infty} \frac{1}{n !} \hbar^{2 g-2+n} F_{g, n}^{H}(t, \ldots, t)\right) .
$$

Then we have

$$
\left[\frac{1}{2} \frac{\partial^{2}}{\partial w^{2}}+\left(\frac{1}{2}+\frac{1}{\hbar}\right) \frac{\partial}{\partial w}-\frac{\partial}{\partial \hbar}\right] Z^{H}(t, \hbar)=0 .
$$

Remark 5.4. Eq. 5.32 is a heat equation, where $\hbar$ is considered as the time variable of the heat conduction. It determines the solution uniquely with the "initial condition"

$$
\left.Z^{H}(t(w), \hbar)\right|_{\hbar \sim 0}=\exp \left(\frac{1}{\hbar} S_{0}^{H}+S_{1}^{H}\right)
$$

given by (5.27) and $(5.28)$.

Proof. Let

$$
F^{H}(t, \hbar)=\sum_{m=0}^{\infty} S_{m}^{H} \hbar^{m-1}=\sum_{g=0}^{\infty} \sum_{n=1}^{\infty} \frac{1}{n !} \hbar^{2 g-2+n} F_{g, n}^{H}(t, \ldots, t) .
$$

In terms of $F^{H}, 5.32$ is equivalent to

$$
\frac{\hbar}{2} \frac{\partial^{2} F^{H}}{\partial w^{2}}+\frac{\hbar}{2}\left(\frac{\partial F^{H}}{\partial w}\right)^{2}+\left[\left(1+\frac{\hbar}{2}\right) \frac{\partial}{\partial w}-\hbar \frac{\partial}{\partial \hbar}\right] F^{H}=0
$$

We apply the operation

$$
\sum_{m=0}^{\infty} \hbar^{m}
$$

to (5.24). The left-hand side is

$$
\sum_{m=0}^{\infty} \hbar^{m}\left(-m+\frac{d}{d w}\right) S_{m+1}^{H}=\left(\frac{\partial}{\partial w}-\hbar \frac{\partial}{\partial \hbar}\right)\left(F^{H}-\frac{1}{\hbar} S_{0}^{H}\right) .
$$

The right-hand side gives

$$
\begin{aligned}
-\frac{\hbar}{2}\left(\frac{\partial^{2}}{\partial w^{2}} \sum_{m=0}^{\infty} S_{m}^{H} \hbar^{m-1}\right. & \left.+\sum_{m=0}^{\infty} \sum_{a+b=m+1} \frac{\partial S_{a}^{H}}{\partial w} \hbar^{a-1} \cdot \frac{\partial S_{b}^{H}}{\partial w} \hbar^{b-1}+\frac{\partial}{\partial w} \sum_{m=0}^{\infty} S_{m}^{H} \hbar^{m-1}\right) \\
& =-\frac{\hbar}{2}\left(\frac{\partial^{2}}{\partial w^{2}} F^{H}+\left(\frac{\partial F^{H}}{\partial w}\right)^{2}-\frac{1}{\hbar^{2}}\left(\frac{\partial S_{0}^{H}}{\partial w}\right)^{2}+\frac{\partial}{\partial w} F^{H}\right) .
\end{aligned}
$$

If we collect all terms that contain $S_{0}^{H}(t)$ in (5.34) and (5.35), we have an equation

$$
\left(t^{2}(t-1) \frac{\partial}{\partial t}+\hbar \frac{\partial}{\partial \hbar}\right) \frac{1}{\hbar} S_{0}^{H}=\frac{1}{2 \hbar}\left(t^{2}(t-1) \frac{\partial}{\partial t} S_{0}^{H}\right)^{2},
$$

or equivalently,

$$
S_{0}^{H}(t)=t^{2}(t-1) \frac{d}{d t} S_{0}^{H}(t)-\frac{1}{2}\left(t^{2}(t-1)\right)^{2}\left(\frac{d S_{0}^{H}(t)}{d t}\right)^{2} .
$$

We see that (5.27) is a solution to (5.36). After eliminating $(5.36)$ from $(5.34)=(5.35)$, we obtain (5.33). This completes the proof. 


\section{The Schur function expansion of the Hurwitz partition function}

Let us introduce the free energy of single Hurwitz numbers by a formal sum as

$$
\begin{aligned}
F^{H}\left(t_{1}, t_{2}, t_{3}, \ldots ; \hbar\right) & =\sum_{g \geq 0, n \geq 1} \frac{1}{n !} \hbar^{2 g-2+n} F_{g, n}^{H}\left(t_{1}, \ldots, t_{n}\right) \\
& =\sum_{g \geq 0, n \geq 1} \frac{1}{n !} \hbar^{2 g-2+n} \sum_{\left(\mu_{1} \ldots, \mu_{n}\right) \in \mathbb{Z}_{+}^{n}} H_{g, n}\left(\mu_{1} \ldots, \mu_{n}\right) e^{-\left(\mu_{1}+\cdots+\mu_{n}\right)} \prod_{i=1}^{n} x_{i}^{\mu_{i}} .
\end{aligned}
$$

The partition function we considered in Section 5 is the principal specialization

$$
Z^{H}(t, \hbar)=\exp \left(F^{H}(t, t, \cdots: \hbar)\right) .
$$

Recall another generating function of the Hurwitz numbers [41, 42, 67] defined by

$$
\begin{gathered}
\mathbf{H}(s, \mathbf{p})=\sum_{g \geq 0, n \geq 1} \mathbf{H}_{g, n}(s, \mathbf{p}), \\
\mathbf{H}_{g, n}(s, \mathbf{p})=\frac{1}{n !} \sum_{\vec{\mu} \in \mathbb{Z}_{+}^{n}} H_{g, n}(\vec{\mu}) \mathbf{p}_{\mu} s^{r(g, \mu)},
\end{gathered}
$$

where $\mathbf{p}_{\mu}=p_{\mu_{1}} \cdots p_{\mu_{n}}$, and

$$
r=r(g, \mu)=2 g-2+n+\sum_{i=1}^{n} \mu_{i}
$$

is again the number of simple ramification point of a Hurwitz cover of genus $g$ and profile $\mu$.

At this point we wish to go back and forth between the following two distinct points of view: One is to regard $\vec{\mu}=\left(\mu_{1}, \ldots, \mu_{n}\right)$ as a vector consisting of positive integers, and the other is to view $\mu$ as a partition of length $n$. For any function $f(\vec{\mu})$ in $\vec{\mu}$ as a vector, we have a change of summation formula

$$
\sum_{\vec{\mu} \in \mathbb{Z}_{+}^{n}} f(\vec{\mu})=\sum_{\mu: \ell(\mu)=n} \frac{1}{|\operatorname{Aut}(\mu)|} \sum_{\sigma \in S_{n}} f\left(\vec{\mu}_{\sigma}\right) .
$$

Here the first sum in the right-hand side runs over partitions $\mu$ of a fixed length $n$, the second sum is over the symmetric group $S_{n}$ of $n$ letters,

$$
\vec{\mu}_{\sigma}=\left(\mu_{\sigma(1)}, \ldots, \mu_{\sigma(n)}\right) \in \mathbb{Z}_{+}^{n}
$$

is the integer vector obtained by permuting the parts of $\mu$ by $\sigma \in S_{n}$, and $\operatorname{Aut}(\mu)$ is the permutation group interchanging the equal parts of $\mu$. As a partition, the length of $\mu$ is denoted by $\ell(\mu)$, and its size by

$$
|\mu|=\sum_{i=1}^{\ell(\mu)} \mu_{i}
$$

Often single Hurwitz numbers are labeled by the genus $g$ and a partition $\mu$. In this case the expression

$$
h_{g, \mu}=\frac{r(g, \mu) !}{|\operatorname{Aut}(\mu)|} H_{g, \ell(\mu)}\left(\mu_{1}, \ldots, \mu_{\ell(\mu)}\right)
$$


is used in the literature, when we do not label the poles, but label the simple ramification points. The generating function then has an expression in terms of sumes over partitions:

$$
\mathbf{H}(s, \mathbf{p})=\sum_{g=0}^{\infty} \sum_{\mu} h_{g, \mu} \mathbf{p}_{\mu} \frac{s^{r(g, \mu)}}{r(g, \mu) !} .
$$

In terms of the ELSV formula $(5.2)$ we have

$$
\begin{aligned}
& \mathbf{H}(s, \mathbf{p}) \\
& \quad=\sum_{g \geq 0, n \geq 1} \frac{1}{n !} s^{2 g-2+n} \sum_{k_{1}+\cdots+k_{n} \leq 3 g-3+n}\left\langle\tau_{k_{1}} \cdots \tau_{k_{n}} \Lambda_{g}^{\vee}(1)\right\rangle_{g, n} \prod_{i=1}^{n} \sum_{\mu_{i}=1}^{\infty} \frac{\mu_{i}^{\mu_{i}+k_{i}}}{\mu_{i} !} s^{\mu_{i}} p_{\mu_{i}}
\end{aligned}
$$

with an appropriate incorporation of (5.5) and (5.6). Recall the Laplace transform (5.7) here for comparison that is assembled into the free energy

$$
\begin{aligned}
F^{H}\left(t_{1}, t_{2} \ldots ; \hbar\right) & :=\sum_{g \geq 0, n \geq 1} \frac{1}{n !} \hbar^{2 g-2+n} F_{g, n}^{H}\left(t_{1}, \ldots, t_{n}\right) \\
& =\sum_{g \geq 0, n \geq 1} \frac{1}{n !} \hbar^{2 g-2+n} \sum_{k_{1}+\cdots+k_{n} \leq 3 g-3+n}\left\langle\tau_{k_{1}} \cdots \tau_{k_{n}} \Lambda_{g}^{\vee}(1)\right\rangle_{g, n} \prod_{i=1}^{n} \sum_{\mu_{i}=1}^{\infty} \frac{\mu_{i}^{\mu_{i}+k_{i}}}{\mu_{i} !} x_{i}^{\mu_{i}} .
\end{aligned}
$$

It is easy to see that the relation between the two sets of variables is exactly the power-sum symmetric functions. Let us re-scale the usual power-sum symmetric function $p_{j}$ of degree $j$ in $x_{i}$ 's with a scale parameter $s$ as follows:

$$
p_{j}(s):=s^{-j}\left(x_{1}^{j}+x_{2}^{j}+x_{3}^{j}+\cdots\right) .
$$

Here we consider $p_{j}(s)$ as a degree $j$ polynomial defined on $\mathbb{C}^{n}$, but the dimension $n$ is unspecified. Then for every $\mu \in \mathbb{Z}_{+}^{n}$, we have

$$
d_{1} \cdots d_{n} \mathbf{p}_{\mu}(s)=s^{-\left(\mu_{1}+\cdots+\mu_{n}\right)}\left(\sum_{\sigma \in S_{n}} \prod_{i=1}^{n} \mu_{i} x_{\sigma(i)}^{\mu_{i}-1}\right) d x_{1} \cdots d x_{n}
$$

as a differential form on $\mathbb{C}^{n}$.

Now from (6.7), 6.8) and (6.4), we obtain

$$
\begin{gathered}
d_{1} \cdots d_{n} \mathbf{H}_{g, n}(s, \mathbf{p}(s))=\sum_{\mu: \ell(\mu)=n} h_{g, \mu} d_{1} \cdots d_{n} \mathbf{p}_{\mu}(s) \frac{s^{r}}{r !} \\
=\sum_{\mu: \ell(\mu)=n} h_{g, \mu} \frac{s^{r}}{r !} s^{-|\mu|} \sum_{\sigma \in S_{n}} \prod_{i=1}^{n} \mu_{i} x_{\sigma(i)}^{\mu_{i}-1} d x_{1} \cdots d x_{n} \\
=\sum_{\mu: \ell(\mu)=n} \frac{1}{|\operatorname{Aut}(\mu)|} H_{g, n}\left(\mu_{1}, \ldots, \mu_{n}\right) s^{2 g-2+n} \sum_{\sigma \in S_{n}} \prod_{i=1}^{n} \mu_{i} x_{\sigma(i)}^{\mu_{i}-1} d x_{1} \cdots d x_{n} \\
=\sum_{\mu \in \mathbb{Z}_{+}^{n}} H_{g, n}\left(\mu_{1}, \ldots, \mu_{n}\right) s^{2 g-2+n} \prod_{i=1}^{n} \mu_{i} x_{i}^{\mu_{i}-1} d x_{1} \cdots d x_{n} \\
=s^{2 g-2+n} d_{1} \cdots d_{n} \sum_{\mu \in \mathbb{Z}_{+}^{n}} H_{g, n}\left(\mu_{1}, \ldots, \mu_{n}\right) \prod_{i=1}^{n} x_{i}^{\mu_{i}}
\end{gathered}
$$




$$
=s^{2 g-2+n} d_{1} \cdots d_{n} F_{g, n}^{H}\left(t_{1}, \ldots, t_{n}\right)=s^{2 g-2+n} W_{g, n}^{H}\left(t_{1}, \ldots, t_{n}\right) .
$$

This formula tells us that the Eynard-Orantin differential form $W_{g, n}^{H}$ is the exterior derivative of $\mathbf{H}_{g, n}(s, \mathbf{p}(s))$ with the identification 6.7). Moreover, we have

$$
s^{2 g-2+n} F_{g, n}^{H}\left(t_{1}, \ldots, t_{n}\right) \equiv \mathbf{H}_{g, n}(s, \mathbf{p}(s)) \bmod \operatorname{Ker}\left(d_{1} \cdots d_{n}\right)
$$

as functions on $\mathbb{C}^{n}$.

Remark 6.1. Let us examine (6.10). For $n=1$, the power sum (6.7) contains only one term and we have

$$
\mathbf{H}_{g, 1}(s, \mathbf{p}(s))=s^{2 g-1} \sum_{k=1}^{\infty} H_{g, 1}(k) p_{k}(s) s^{k}=s^{2 g-1} \sum_{k=1}^{\infty} H_{g, 1}(k) x^{k}=s^{2 g-1} F_{g, 1}^{H}\left(t_{1}\right) .
$$

Thus 6.10 is an equality for $n=1$. In general what happens is

$$
\begin{aligned}
\mathbf{H}_{g, n}( & s, \mathbf{p})=\frac{1}{n !} s^{2 g-2+n} \sum_{\vec{\mu} \in \mathbb{Z}_{+}^{n}} H_{g, n}(\vec{\mu}) \prod_{i=1}^{n}\left(x_{1}^{\mu_{1}}+x_{2}^{\mu_{2}}+\cdots+x_{n}^{\mu_{n}}\right) \\
& =s^{2 g-2+n} \sum_{\vec{\mu} \in \mathbb{Z}_{+}^{n}} H_{g, n}(\vec{\mu}) x_{1}^{\mu_{1}} x_{2}^{\mu_{2}} \cdots x_{n}^{\mu_{n}}+(\text { terms with less than } n \text { variables }) .
\end{aligned}
$$

Therefore, 6.10 is never an equality for $n>1$.

However, the principal specialization $t=t_{1}=t_{2}=t_{3}=\cdots$ corresponds to evaluating

$$
p_{j}=\left(\frac{x}{s}\right)^{j} .
$$

With this identification we have again an equality

$$
\begin{aligned}
& \mathbf{H}(s, \mathbf{p}) \\
& =\sum_{g \geq 0, n \geq 1} \frac{1}{n !} s^{2 g-2+n} \sum_{\vec{\mu} \in \mathbb{Z}_{+}^{n}} H_{g, n}(\vec{\mu}) x^{\left(\mu_{1}+\cdots+\mu_{n}\right)}=\sum_{g \geq 0, n \geq 1} \frac{1}{n !} s^{2 g-2+n} F_{g, n}^{H}(t, t, \ldots, t) .
\end{aligned}
$$

In Section 5 we noted that the Eynard-Orantin recursion for Hurwitz numbers is the Laplace transform of the cut-and-join equation (5.21) [26, 60]. Another consequence of the same combinatorial equation is a heat equation [35, 41, 79.

$$
\frac{\partial}{\partial s} e^{\mathbf{H}(s, \mathbf{p})}=\frac{1}{2}\left[\sum_{i, j \geq 1}\left((i+j) p_{i} p_{j} \frac{\partial}{\partial p_{i+j}}+i j p_{i+j} \frac{\partial^{2}}{\partial p_{i} \partial p_{j}}\right)\right] e^{\mathbf{H}(s, \mathbf{p})},
$$

with the initial condition $\mathbf{H}(0, \mathbf{p})=p_{1}$. An important and fundamental fact here is that the heat equation (6.15) implies that $e^{\mathbf{H}(s, \mathbf{p})}$ is a KP $\tau$-function for each value of $s$ [41, 42, 67, 79, . Let us recall this fact here. We note that a solution of the heat equation is expanded by the eigenfunction of the second order operator. In our case of (6.15), the eigenfunctions of the cut-and-join operator on the right-hand side are given by the Schur functions.

For a partition $\mu=\left(\mu_{1} \geq \mu_{2} \geq \cdots\right)$ of a finite length $\ell(\mu)$, we define the shifted power-sum function by

$$
\mathbf{p}_{r}[\mu]:=\sum_{i=1}^{\infty}\left[\left(\mu_{i}-i+\frac{1}{2}\right)^{r}-\left(-i+\frac{1}{2}\right)^{r}\right] .
$$


This is a finite sum of $\ell(\mu)$ terms. In this paper we consider $\mathbf{p}_{r}[\mu]$ as a number associated with a partition $\mu$. Then we have [35, 79]

$$
\sum_{i, j \geq 1}\left((i+j) p_{i} p_{j} \frac{\partial}{\partial p_{i+j}}+i j p_{i+j} \frac{\partial^{2}}{\partial p_{i} \partial p_{j}}\right) s_{\mu}(\mathbf{p})=\mathbf{p}_{2}[\mu] \cdot s_{\mu}(\mathbf{p}),
$$

where $s_{\mu}(\mathbf{p})$ is the Schur function defined by

$$
\begin{aligned}
s_{\mu}(\mathbf{p}) & =\sum_{|\lambda|=|\mu|} \frac{\chi_{\mu}(\lambda)}{z_{\lambda}} \mathbf{p}_{\lambda}, \\
z_{\mu} & =\prod_{i=1}^{\ell(\mu)} m_{i} ! i^{m_{i}}, \\
m_{i} & =\text { the number of parts in } \mu \text { of length } i,
\end{aligned}
$$

and $\chi_{\mu}(\lambda)$ is the value of the irreducible character of the representation $\mu$ of the symmetric group evaluated at the conjugacy class $\lambda$. If we write

$$
\triangle=\frac{1}{2} \sum_{i, j \geq 1}\left((i+j) p_{i} p_{j} \frac{\partial}{\partial p_{i+j}}+i j p_{i+j} \frac{\partial^{2}}{\partial p_{i} \partial p_{j}}\right),
$$

Then

$$
\triangle s_{\mu}(\mathbf{p})=\frac{1}{2} \mathbf{p}_{2}[\mu] \cdot s_{\mu}(\mathbf{p})
$$

and

$$
\frac{\partial}{\partial s} e^{\mathbf{H}(s, \mathbf{p})}=\Delta e^{\mathbf{H}(s, \mathbf{p})} .
$$

Therefore, we have an expansion formula

$$
e^{\mathbf{H}(s, \mathbf{p})}=\sum_{\mu} c_{\mu} s_{\mu}(\mathbf{p}) e^{\frac{1}{2} \mathbf{p}_{2}[\mu] s}
$$

for a constant $c_{\mu}$ associated with every partition $\mu$. The constants are determined by the initial value. Since the initial condition is

$$
\left.e^{\mathbf{H}(s, \mathbf{p})}\right|_{s=0}=e^{p_{1}}=\sum_{\mu} c_{\mu} s_{\mu}(\mathbf{p}),
$$

we conclude that

$$
c_{\mu}=s_{\mu}(1,0,0, \ldots, 0) .
$$

This follows from the Cauchy identity

$$
\frac{1}{\prod_{i, j}\left(1-x_{i} y_{j}\right)}=\sum_{\mu} s_{\mu}(\mathbf{p}) s_{\mu}\left(\mathbf{p}^{y}\right),
$$

where

$$
p_{j}=\sum_{i} x_{i}^{j} \quad \text { and } \quad p_{j}^{y}=\sum_{i} y_{i}^{j} .
$$

Since we have

$$
\sum_{\mu} s_{\mu}(\mathbf{p}) s_{\mu}\left(\mathbf{p}^{y}\right)=\frac{1}{\prod_{i, j}\left(1-x_{i} y_{j}\right)}=\exp \left(-\sum_{i, j} \log \left(1-x_{i} y_{j}\right)\right)
$$




$$
=\exp \left(\sum_{i, j} \sum_{m \geq 1} \frac{1}{m} x_{j}^{m} y_{i}^{m}\right)=\exp \left(\sum_{m \geq 1} \frac{1}{m} p_{m} p_{m}^{y}\right),
$$

the restriction of 6.19 to $p_{1}^{y}=1$ and $p_{m}^{y}=0$ for all $m \geq 2$ reduces to

$$
e^{p_{1}}=\sum_{\mu} s_{\mu}(1,0, \ldots, 0) s_{\mu}(\mathbf{p}) .
$$

Because of the determinantal formula for the Schur functions, $s_{\mu}(1,0,0, \ldots, 0)$ 's are the Plücker coordinate of a point of the Sato Grassmannian. It follows that

$$
s_{\mu}(1,0,0, \ldots, 0) e^{\frac{1}{2} \mathbf{p}_{2}[\mu] s}
$$

for all $\mu$ also form the Plücker coordinates because of $(6.16)$. Then by a theorem of Sato [73,

$$
e^{\mathbf{H}(s, \mathbf{p})}=\sum_{\mu} s_{\mu}(1,0,0, \ldots, 0) s_{\mu}(\mathbf{p}) e^{\frac{1}{2} \mathbf{p}_{2}[\mu] s}=\sum_{\mu} \frac{\operatorname{dim} \mu}{|\mu| !} e^{\frac{1}{2} \mathbf{p}_{2}[\mu] s} s_{\mu}(\mathbf{p})
$$

is a $\tau$-function of the $\mathrm{KP}$ equations. Here $\operatorname{dim} \mu$ is the dimension of the irreducible representation of the symmetric group $S_{|\mu|}$ belonging to the partition $\mu$.

Thus we have established

Theorem 6.2. The Hurwitz partition function $Z^{H}(t, \hbar)$ of (5.31) is obtained by evaluation of the KP $\tau$-function $e^{\mathbf{H}(s, \mathbf{p})}$ at $(s, \mathbf{p})=(\hbar, \mathbf{p}(\hbar))$ :

$$
Z^{H}(t, \hbar)=e^{\mathbf{H}(\hbar, \mathbf{p}(\hbar))} .
$$

Here $\mathbf{p}(\hbar)$ means the principal specialization

$$
p_{j}=\left(\frac{x}{\hbar}\right)^{j}
$$

for every $j=1,2,3, \ldots$ The $t$-variable and the $x$-variable are related by

$$
x=\frac{t-1}{t} e^{\frac{1}{t}-1} .
$$

Since we have a concrete expansion formula 6.20 for $e^{\mathbf{H}(s, \mathbf{p})}$, it is straightforward to find a formula for its principal specialization. Let us look at (6.19) again. This time we apply the principal specialization to both $\mathbf{p}$ and $\mathbf{p}^{y}$, meaning that we substitute

$$
p_{m}=x^{m} \quad \text { and } \quad p_{m}^{y}=y^{m} .
$$

Then we have

$$
\sum_{\mu} s_{\mu}(\mathbf{p}) s_{\mu}\left(\mathbf{p}^{y}\right)=\sum_{m=0}^{\infty} x^{m} y^{m}
$$

after the double principal specialization. Therefore, the sum with respect to all partitions $\mu$ is reduced to the sum with respect to only one-part partitions, i.e., $\mu=(m)$. All other partitions contribute 0 . Thus $s_{\mu}=s_{m}=h_{m}$, which is the $m$-th complete symmetric function. But because of the principal specialization, we simply have $h_{m}=x^{m}$. We note that if $\mu=(m)$, then 6.16 reduces to

$$
\mathbf{p}_{2}[(m)]=\left(m-1+\frac{1}{2}\right)^{2}-\left(-1+\frac{1}{2}\right)^{2}=m(m-1) .
$$


We therefore conclude that

$$
Z^{H}(t, \hbar)=e^{\mathbf{H}(\hbar, \mathbf{p}(\hbar))}=\sum_{m=0}^{\infty} \frac{1}{m !} e^{\frac{1}{2} m(m-1) \hbar}\left(\frac{x}{\hbar}\right)^{m} .
$$

We have now established a theorem of Zhou [82].

Theorem 6.3 (82]). The same Hurwitz partition function satisfies a differential-difference equation

$$
\left(\hbar \frac{\partial}{\partial w}+e^{-w} e^{-\hbar \frac{\partial}{\partial w}}\right) Z^{H}(t, \hbar)=0 .
$$

Here again $t$ is a function in $w$, which is given by

$$
t=1+\sum_{k=1}^{\infty} \frac{k^{k}}{k !} x^{k}=1+\sum_{k=1}^{\infty} \frac{k^{k}}{k !} e^{-w k}
$$

The characteristic variety of this equation, with the identification of

$$
z=-\hbar \frac{\partial}{\partial w}
$$

is the Lagrangian immersion $e^{-w}=z e^{-z}$.

Proof. Let us denote

$$
a_{m}=e^{\frac{1}{2} m(m-1) \hbar}\left(\frac{x}{\hbar}\right)^{m}=e^{(1+2+\cdots+(m-1)) \hbar}\left(\frac{x}{\hbar}\right)^{m} .
$$

Then

$$
Z^{H}(t, \hbar)=\sum_{m=0}^{\infty} \frac{1}{m !} a_{m}, \quad a_{m+1}=e^{m \hbar} a_{m} \frac{x}{\hbar}, \quad \text { and } \quad x \frac{d}{d x} a_{m}=m a_{m} .
$$

We note that $x \frac{d}{d x}$ operates as the multiplication of $m$ to $a_{m}$. Therefore,

$$
\begin{aligned}
-\hbar \frac{\partial}{\partial w} Z^{H}(t, \hbar)=\hbar x \frac{d}{d x} \sum_{m=0}^{\infty} \frac{1}{m !} a_{m}=\hbar \sum_{m=0}^{\infty} \frac{1}{m !} & a_{m+1}=x \sum_{m=0}^{\infty} \frac{1}{m !} e^{m \hbar} a_{m} \\
& =x e^{\hbar x \frac{d}{d x}} Z^{H}(t, \hbar)=e^{-w} e^{-\hbar \frac{\partial}{\partial w}} Z^{H}(t, \hbar) .
\end{aligned}
$$

This completes the proof.

Remark 6.4. The asymptotic behavior of $Z^{H}(t, \hbar)$ near $\hbar=0$ is determined by

$$
\exp \left(\frac{1}{\hbar} S_{0}^{H}+S_{1}^{H}\right)
$$

From 6.24) we have

$$
\begin{gathered}
0=\left.\exp \left(-\frac{1}{\hbar} S_{0}^{H}-S_{1}^{H}\right)\left(\hbar \frac{d}{d w}+e^{-w} e^{-\hbar \frac{d}{d w}}\right) \exp \left(\frac{1}{\hbar} S_{0}^{H}+S_{1}^{H}\right)\right|_{\hbar=0} \\
=\frac{d}{d w} S_{0}^{H}(t(w))+\left.e^{-w} \exp \left(\frac{S_{0}^{H}(t(w-\hbar))-S_{0}^{H}(t(w))}{\hbar}\right)\right|_{\hbar=0} \\
=\frac{d}{d w} S_{0}^{H}+e^{-w} e^{-\frac{d}{d w} S_{0}^{H}}=-z+x e^{z} .
\end{gathered}
$$

We thus recover the Lagrangian immersion in this way as well. 
Remark 6.5. We can directly verify that the expression $(6.23)$ of $Z^{H}(t, \hbar)$ satisfies the Schrödinger equation (5.32). Indeed, since $x=e^{-w}$,

$$
\begin{aligned}
{\left[\frac{1}{2} \frac{\partial^{2}}{\partial w^{2}}+\right.} & \left.\left(\frac{1}{2}+\frac{1}{\hbar}\right) \frac{\partial}{\partial w}-\frac{\partial}{\partial \hbar}\right] \sum_{m=0}^{\infty} \frac{1}{m !} e^{\frac{1}{2} m(m-1) \hbar} \frac{1}{\hbar^{m}} e^{-m w} \\
& =\sum_{m=0}^{\infty} \frac{1}{m !} e^{\frac{1}{2} m(m-1) \hbar} \frac{1}{\hbar^{m}} e^{-m w}\left[\frac{1}{2} m^{2}-m\left(\frac{1}{2}+\frac{1}{\hbar}\right)-\frac{1}{2} m(m-1)+\frac{m}{\hbar}\right]=0 .
\end{aligned}
$$

\section{Conclusion}

The main purpose of this paper is to derive the Schrödinger equation of the partition function from the integrated Eynard-Orantin topological recursion, when there is an Amodel counting problem whose mirror dual is the Eynard-Orantin theory. We examined two different types of counting problem of ramified covering of $\mathbb{P}^{1}$ : one is Grothendieck's dessins d'enfants, and the other single Hurwitz numbers. The first example leads to a Lagrangian immersion in $T^{*} \mathbb{C}$ defined by a Laurent polynomial equation (3.6), while the latter corresponds to the Lambert curve in $T^{*} \mathbb{C}^{*}$ given by an exponential equation (5.16).

If we start with the Eynard-Orantin recursion (2.6) and define the primitive functions $F_{g, n}$ by (2.12), then we have the ambiguity in the constants of integration. However, if we start with an A-model, then the primitive functions $F_{g, n}$ 's are given by the Laplace transform of the solution to the A-model problem. The examples we have studied in this paper show that always there is a natural zero $t_{i}=a$ of $F_{g, n}\left(t_{1}, \ldots, t_{n}\right)$ in each variable, such as 4.2 and (5.13), when $2 g-2+n>0$. Thus the integration formula

$$
F_{g, n}\left(t_{1}, \ldots, t_{n}\right)=\int_{a}^{t_{1}} \cdots \int_{a}^{t_{n}} W_{g, n}\left(t_{1}, \ldots, t_{n}\right)
$$

uniquely determines $F_{g, n}$ from $W_{g, n}$. The integral transform equation $(2.6)$ for $W_{g, n}$ is then equivalent to a differential transform equation for $F_{g, n}$, such as 4.3 and $(5.22$ for our examples. Because of our assumption for the Lagrangian immersion that the Lagrangian singularities are simply ramified, the differential recursion equation for $F_{g, n}$ is expected to be a second order PDE. Then by taking the principal specialization, we obtain a second order differential equation in $t$ and $\hbar$. For our examples we have thus established (4.8) and 5.32 .

Although (4.8) is holonomic if we consider $\hbar$ a constant, (5.32) is a PDE containing the $\hbar$-differentiation as well. Therefore, it is not holonomic. This difference comes from the constant term $2 g-2+n$ in the differential operator of the right-hand side of (5.22). After taking the $n$-fold symmetric exterior differentiation, this term drops, and thus the EynardOrantin recursion for Hurwitz numbers [26] takes the same shape as that of the Catalan case (3.10).

With the analysis of our examples, we notice that the issue of the constants of integration in 2.12) is not a simple matter. Only the corresponding A-model can dictate which constants of integration we should choose. Otherwise, the Schrödinger equation we wish to establish would take a totally different shape, depending on the choice of the constants.

Our second equation (6.24) for Hurwitz numbers is much similar to (4.8) in many ways, such as it is holonomic for each fixed $\hbar$. But this differential-difference equation is not a direct consequence of the differential equation (5.22), while it recovers the Lagrangian immersion more directly than $(5.32)$. We also note here the commutator relation $[P, Q]=P$ 
of [49] that we mentioned in Introduction. We refer to [49] for a further integrable system theoretic analysis of these equations.

Interestingly, we derive (6.24) from the fact that there is another generating function for single Hurwitz numbers, which admits a Schur function expansion. This last point is a more general feature. In [59] we discover that there is a generalization of (6.24) for the case of double Hurwitz numbers and $r$-spin structures, which reduces to (6.24) as a special case. A detailed analysis of double Hurwitz numbers, or orbifold Hurwitz numbers, will be given elsewhere.

So far all these examples have a genus 0 spectral curve. Hence the proposed algebraic K-theory obstruction of Gukov and Sułkowski [39] automatically vanishes. It is also pointed out by Borot and Eynard [6] that, for a higher genus spectral curve, the definition of the partition function of the B-model needs to be modified, by including a theta function factor known as a non-perturbative sector. This modification also assures the modular invariance of the partition function. A further investigation is awaited here.

The examples we have carried out in this paper suggest that the expected Schrödinger equations for the knot A-polynomials should form a rather special class of the general Eynard-Orantin mechanism. This seems to be due to the integer-coefficient Laurent polynomial expression of the A-polynomials, and their $K_{2}$ Lagrangian property of Kontsevich.

\section{Appendix A. Proof of the Schrödinger equation for the Catalan case}

In this Appendix we give the proof of Theorem 4.5. In Section 4 we reduced the proof to verifying the recursion formula 4.16 . To prove this, we need the following trivial lemma.

Lemma A.1. Let $f\left(t_{1}, \ldots, t_{n}\right)$ be a symmetric function in $n$ variables. Then

$$
\begin{aligned}
\frac{d}{d t} f(t, t, \ldots, t)=n & {\left.\left[\frac{\partial}{\partial u} f(u, t, \ldots, t)\right]\right|_{u=t} ; } \\
\frac{d^{2}}{d t^{2}} f(t, t, \ldots, t)=n & {\left.\left[\frac{\partial^{2}}{\partial u^{2}} f(u, t, \ldots, t)\right]\right|_{u=t} } \\
& +\left.n(n-1)\left[\frac{\partial^{2}}{\partial u_{1} \partial u_{2}} f\left(u_{1}, u_{2}, t, \ldots, t\right)\right]\right|_{u_{1}=u_{2}=t}
\end{aligned}
$$

For two functions in one variable $g(x)$ and $f(x)$, we have

$$
\left.\left[\frac{1}{x-y}\left(g(x) \frac{d f(x)}{d x}-g(y) \frac{d f(y)}{d y}\right)\right]\right|_{x=y}=g^{\prime}(x) f^{\prime}(x)+g(x) f^{\prime \prime}(x) .
$$

Proof. For any function $f$ we have

$$
\frac{d}{d t} f(t, t, \ldots, t)=\left.\left[\sum_{j=1}^{n} \frac{\partial}{\partial t_{j}} f\left(t_{1}, t_{2}, \ldots, t_{n}\right)\right]\right|_{t_{1}=t_{2}=\cdots=t_{n}=t} .
$$

Therefore,

$$
\begin{gathered}
\frac{d^{2}}{d t^{2}} f(t, t, \ldots, t)=\left.\left[\left(\sum_{j=1}^{n} \frac{\partial}{\partial t_{j}}\right)^{2} f\left(t_{1}, t_{2}, \ldots, t_{n}\right)\right]\right|_{t_{1}=t_{2}=\cdots=t_{n}=t} \\
=\left.\left[\left(\sum_{j=1}^{n} \frac{\partial^{2}}{\partial t_{j}^{2}}+2 \sum_{i \neq j} \frac{\partial^{2}}{\partial t_{i} \partial t_{j}}\right) f\left(t_{1}, t_{2}, \ldots, t_{n}\right)\right]\right|_{t_{1}=t_{2}=\cdots=t_{n}=t} .
\end{gathered}
$$


Eq. A.1 holds when $f$ is symmetric. The second equation A.2 follows from l'Hôpital's rule.

Now we are ready to give the proof of the recursion (4.16).

Proof of (4.16). The left-hand side of 4.16) is

$$
\frac{d}{d t} S_{m+1}=\sum_{2 g-2+n=m} \frac{1}{n !} \frac{d}{d t} F_{g, n}(t, \ldots, t)=\left.\sum_{2 g-2+n=m} \frac{1}{(n-1) !}\left(\frac{\partial}{\partial t_{1}} F_{g, n}\left(t_{1}, t, \ldots, t\right)\right)\right|_{t_{1}=t} .
$$

Thus we apply the operation

$$
\sum_{2 g-2+n=m} \frac{1}{(n-1) !}
$$

to each line of the right-hand side of $(4.3)$ and set all variables equal to $t$.

From Line 1 of the right-hand side of (4.3), we have

$$
\begin{aligned}
& -\frac{1}{16} \sum_{2 g-2+n=m} \frac{1}{(n-1) !} \sum_{j=2}^{n} \frac{t_{j}}{\left(t_{1}+t_{j}\right)\left(t_{1}-t_{j}\right)} \\
& \times\left.\left(\frac{\left(t_{1}^{2}-1\right)^{3}}{t_{1}^{2}} \frac{\partial}{\partial t_{1}} F_{g, n-1}^{C}\left(t_{[\hat{j}]}\right)-\frac{\left(t_{j}^{2}-1\right)^{3}}{t_{j}^{2}} \frac{\partial}{\partial t_{j}} F_{g, n-1}^{C}\left(t_{[\hat{1}]}\right)\right)\right|_{t_{1}=\cdots=t_{n}=t} \\
& =-\frac{1}{32} \sum_{2 g-2+n=m} \frac{1}{(n-2) !}\left[\left(\frac{d}{d t} \frac{\left(t^{2}-1\right)^{3}}{t^{2}}\right) \frac{\partial}{\partial t_{1}} F_{g, n-1}^{C}\left(t_{1}, t, \ldots, t\right)\right. \\
& \left.+\frac{\left(t^{2}-1\right)^{3}}{t^{2}} \frac{\partial^{2}}{\partial t_{1}^{2}} F_{g, n-1}^{C}\left(t_{1}, t, \ldots, t\right)\right]\left.\right|_{t_{1}=t} \\
& =-\frac{1}{32}\left(\frac{d}{d t} \frac{\left(t^{2}-1\right)^{3}}{t^{2}}\right) \sum_{2 g-2+n=m} \frac{1}{(n-1) !} \frac{d}{d t} F_{g, n-1}^{C}(t, \ldots, t) \\
& \left.-\frac{1}{32} \frac{\left(t^{2}-1\right)^{3}}{t^{2}} \sum_{2 g-2+n=m} \frac{1}{(n-2) !} \frac{\partial^{2}}{\partial t_{1}^{2}} F_{g, n-1}^{C}\left(t_{1}, t, \ldots, t\right)\right]\left.\right|_{t_{1}=t} \\
& =-\frac{1}{32}\left(\frac{d}{d t} \frac{\left(t^{2}-1\right)^{3}}{t^{2}}\right) \sum_{2 g^{\prime}-2+n^{\prime}=m-1} \frac{1}{n^{\prime} !} \frac{d}{d t} F_{g^{\prime}, n^{\prime}}^{C}(t, \ldots, t) \\
& \left.-\frac{1}{32} \frac{\left(t^{2}-1\right)^{3}}{t^{2}} \sum_{2 g^{\prime}-2+n^{\prime}=m-1} \frac{1}{\left(n^{\prime}-1\right) !} \frac{\partial^{2}}{\partial t_{1}^{2}} F_{g^{\prime}, n^{\prime}}^{C}\left(t_{1}, t, \ldots, t\right)\right]\left.\right|_{t_{1}=t} \\
& =-\frac{1}{32}\left(\frac{d}{d t} \frac{\left(t^{2}-1\right)^{3}}{t^{2}}\right) \frac{d}{d t} S_{m} \\
& \left.-\frac{1}{32} \frac{\left(t^{2}-1\right)^{3}}{t^{2}} \sum_{2 g^{\prime}-2+n^{\prime}=m-1} \frac{1}{\left(n^{\prime}-1\right) !} \frac{\partial^{2}}{\partial t_{1}^{2}} F_{g^{\prime}, n^{\prime}}^{C}\left(t_{1}, t, \ldots, t\right)\right]\left.\right|_{t_{1}=t} .
\end{aligned}
$$

From Line 2 we obtain

$$
-\left.\frac{1}{16} \sum_{2 g-2+n=m} \frac{1}{(n-1) !} \sum_{j=2}^{n} \frac{\left(t_{1}^{2}-1\right)^{2}}{t_{1}^{2}} \frac{\partial}{\partial t_{1}} F_{g, n-1}^{C}\left(t_{[\hat{j}]}\right)\right|_{t_{1}=\cdots=t_{n}=t}
$$




$$
\begin{array}{r}
=-\left.\frac{1}{16} \frac{\left(t^{2}-1\right)^{2}}{t^{2}} \sum_{2 g-2+n=m} \frac{1}{(n-2) !} \frac{\partial}{\partial t_{1}} F_{g, n-1}^{C}\left(t_{1}, t, \ldots, t\right)\right|_{t_{1}=t} \\
=-\frac{1}{16} \frac{\left(t^{2}-1\right)^{2}}{t^{2}} \frac{d}{d t} S_{m} .
\end{array}
$$

Line 3 produces

$$
\begin{aligned}
& -\left.\frac{1}{32} \sum_{2 g-2+n=m} \frac{1}{(n-1) !}\left[\frac{\left(t_{1}^{2}-1\right)^{3}}{t_{1}^{2}} \frac{\partial^{2}}{\partial u_{1} \partial u_{2}} F_{g-1, n+1}^{C}\left(u_{1}, u_{2}, t_{2}, t_{3}, \ldots, t_{n}\right)\right]\right|_{u_{1}=u_{2}=t_{1}=\cdots=t_{n}=t} \\
& =-\left.\frac{1}{32} \frac{\left(t^{2}-1\right)^{3}}{t^{2}} \sum_{2 g-2+n=m} \frac{1}{(n-1) !}\left[\frac{\partial^{2}}{\partial u_{1} \partial u_{2}} F_{g-1, n+1}^{C}\left(u_{1}, u_{2}, t, \ldots, t\right)\right]\right|_{u_{1}=u_{2}=t} \\
& =-\left.\frac{1}{32} \frac{\left(t^{2}-1\right)^{3}}{t^{2}} \sum_{2 g^{\prime}-2+n^{\prime}=m-1, n^{\prime} \geq 2} \frac{1}{\left(n^{\prime}-2\right) !}\left[\frac{\partial^{2}}{\partial u_{1} \partial u_{2}} F_{g^{\prime}, n^{\prime}}^{C}\left(u_{1}, u_{2}, t, \ldots, t\right)\right]\right|_{u_{1}=u_{2}=t} .
\end{aligned}
$$

Finally, Line 4 gives

$$
\begin{aligned}
& -\frac{1}{32} \sum_{2 g-2+n=m} \frac{1}{(n-1) !}\left[\frac{\left(t_{1}^{2}-1\right)^{3}}{t_{1}^{2}}\right. \\
& \left.\times \sum_{\substack{g_{1}+g_{2}=g \\
I \sqcup J=\{2,3, \ldots, n\}}}^{\text {stable }} \frac{\partial}{\partial t_{1}} F_{g_{1},|I|+1}^{C}\left(t_{1}, t_{I}\right) \frac{\partial}{\partial t_{1}} F_{g_{2},|J|+1}^{C}\left(t_{1}, t_{J}\right)\right]\left.\right|_{t_{1}=\cdots=t_{n}=t} \\
& =-\frac{1}{32} \frac{\left(t^{2}-1\right)^{3}}{t^{2}} \sum_{2 g-2+n=m} \frac{1}{(n-1) !} \\
& \times\left.\sum_{\substack{g_{1}+g_{2}=g \\
n_{1}+n_{2}=n-1}}^{\text {stable }}\left[\left(\begin{array}{c}
n-1 \\
n_{1}
\end{array}\right) \frac{\partial}{\partial t_{1}} F_{g_{1}, n_{1}+1}^{C}\left(t_{1}, t, \ldots, t\right) \frac{\partial}{\partial t_{1}} F_{g_{2}, n_{2}+1}^{C}\left(t_{1}, t, \ldots, t\right)\right]\right|_{t_{1}=t} \\
& =-\frac{1}{32} \frac{\left(t^{2}-1\right)^{3}}{t^{2}} \\
& \times\left.\sum_{2 g-2+n=m} \sum_{\substack{g_{1}+g_{2}=g \\
n_{1}+n_{2}=n-1}}^{\text {stable }}\left[\frac{1}{n_{1} !} \frac{\partial}{\partial t_{1}} F_{g_{1}, n_{1}+1}^{C}\left(t_{1}, t, \ldots, t\right) \frac{1}{n_{2} !} \frac{\partial}{\partial t_{1}} F_{g_{2}, n_{2}+1}^{C}\left(t_{1}, t, \ldots, t\right)\right]\right|_{t_{1}=t} \\
& =-\frac{1}{32} \frac{\left(t^{2}-1\right)^{3}}{t^{2}} \\
& \times \sum_{2 g-2+n=m} \sum_{\substack{g_{1}+g_{2}=g \\
n_{1}+n_{2}=n-1}}^{\text {stable }} \frac{1}{\left(n_{1}+1\right) !} \frac{d}{d t} F_{g_{1}, n_{1}+1}^{C}(t, \ldots, t) \frac{1}{\left(n_{2}+1\right) !} \frac{d}{d t} F_{g_{2}, n_{2}+1}^{C}(t, \ldots, t) \\
& =-\frac{1}{32} \frac{\left(t^{2}-1\right)^{3}}{t^{2}} \sum_{\substack{a+b=m+1 \\
a, b \geq 2}} \frac{d S_{a}}{d t} \frac{d S_{b}}{d t} .
\end{aligned}
$$

In the last line we note that unstable geometries $(g, n)=(0,1)$ and $(0,2)$ are included only in $S_{0}$ and $S_{1}$. This line gives the correct contribution of the product term in (4.16). 
Since

$$
-\frac{1}{32}\left(\frac{d}{d t} \frac{\left(t^{2}-1\right)^{3}}{t^{2}}\right)-\frac{1}{16} \frac{\left(t^{2}-1\right)^{2}}{t^{3}}=-\frac{1}{16} \frac{\left(t^{2}-1\right)^{2}}{t^{3}}\left(2 t^{2}+t+1\right),
$$

we have the correct term for $d S_{m} / d t$ in (4.16). The remaining terms are second derivatives, and we calculate

$$
\begin{gathered}
-\left.\frac{1}{32} \frac{\left(t^{2}-1\right)^{3}}{t^{2}} \sum_{2 g^{\prime}-2+n^{\prime}=m-1} \frac{1}{\left(n^{\prime}-1\right) !} \frac{\partial^{2}}{\partial t_{1}^{2}} F_{g^{\prime}, n^{\prime}}^{C}\left(t_{1}, t, \ldots, t\right)\right|_{t_{1}=t} \\
-\left.\frac{1}{32} \frac{\left(t^{2}-1\right)^{3}}{t^{2}} \sum_{2 g^{\prime}-2+n^{\prime}=m-1, n^{\prime} \geq 2} \frac{1}{\left(n^{\prime}-2\right) !}\left[\frac{\partial^{2}}{\partial u_{1} \partial u_{2}} F_{g^{\prime}, n^{\prime}}^{C}\left(u_{1}, u_{2}, t, \ldots, t\right)\right]\right|_{u_{1}=u_{2}=t} \\
=-\frac{1}{32} \frac{\left(t^{2}-1\right)^{3}}{t^{2}} \sum_{2 g^{\prime}-2+n^{\prime}=m-1} \frac{1}{n^{\prime} !} \\
\times\left(\left.n^{\prime} \frac{\partial^{2}}{\partial t_{1}^{2}} F_{g^{\prime}, n^{\prime}}^{C}\left(t_{1}, t, \ldots, t\right)\right|_{t_{1}=t}+\left.n^{\prime}\left(n^{\prime}-1\right)\left[\frac{\partial^{2}}{\partial u_{1} \partial u_{2}} F_{g^{\prime}, n^{\prime}}^{C}\left(u_{1}, u_{2}, t, \ldots, t\right)\right]\right|_{u_{1}=u_{2}=t}\right) \\
=-\frac{1}{32} \frac{\left(t^{2}-1\right)^{3}}{t^{2}} \frac{d^{2}}{d t^{2}} S_{m} .
\end{gathered}
$$

This completes the derivation of (4.16) from (4.3).

\section{Appendix B. Hierarchy of equations for $S_{m}$}

In this paper we proved that the Catalan and Hurwitz partition functions $Z$ satisfy appropriate Schrödinger equations, which can be written as

$$
\widehat{A} Z=0 .
$$

In general such partition functions have an expansion $Z=\exp F=\exp \left(\sum_{m=0}^{\infty} \hbar^{m-1} S_{m}\right)$, where $S_{m}$ are expressed in terms of $F_{g, n}$ as in 4.9 )

$$
S_{m}=\sum_{2 g-2+n=m-1} \frac{1}{n !} F_{g, n}
$$

while $\widehat{A}$ is a differential (or a difference-differential) operator expressed in terms of $x$ and $\hbar \frac{d}{d x}$. Our proofs relied on the knowledge of the recursion equations satisfied by $F_{g, n}$, and we did not need to determine coefficients $S_{m}$ explicitly. However, from the viewpoint of the asymptotic expansion in $\hbar$, these are the coefficients $S_{m}$ which play the fundamental role. They can be determined order by order in $\hbar$ once the form of the operator $\widehat{A}$ is known, or in turn - if the form of $S_{m}$ is known, it allows to determine, also order by order in $\hbar$, the operator $\widehat{A}$ from the knowledge of its symbol $A=A(x, y)$. The relation between $S_{m}$ and $\widehat{A}$ can be encoded in a hierarchy of differential equations which was analyzed in [39]. In what follows we summarize the structure of this hierarchy and use it to determine several coefficients $S_{m}$ in the examples considered in this paper.

As $\widehat{A}$ is an operator expression, we should specify the ordering of $x$ and $\hbar \frac{d}{d x}$ operators it is built from. Let us choose the ordering such that, in each monomial summand, all $\hbar \frac{d}{d x}$ are to the right of $x$. In general, the Schrödinger operator can be written then in the form

$$
\widehat{A}=\widehat{A}_{0}+\hbar \widehat{A}_{1}+\hbar^{2} \widehat{A}_{2}+\ldots,
$$


which reduces in the $\hbar \rightarrow 0$ limit to the symbol $A_{0}=\widehat{A}_{0}=A=A(x, y)$. The examples considered in this paper are in fact quite special - in both Catalan and Hurwitz case all $A_{k}=0$ for $k \geq 1$, and the issue of ordering is irrelevant (because monomials involving both $x$ and $\hbar \frac{d}{d x}$ do not arise), so that the Schrödinger operator can be obtained from the symbol $A_{0}$ just by the substitution $y \rightarrow \hbar \frac{d}{d x}$. Nonetheless, let us recall the most general form of the hierarchy of differential equations which relates a general operator of the form (B.3) to the coefficients $S_{m}$. This hierarchy can be written as [39]

$$
\sum_{r=0}^{n} \mathfrak{D}_{r} A_{n-r}=0,
$$

where $A_{n-r}$ are symbols of the operators $\widehat{A}_{n-r}$, and $\mathfrak{D}_{r}$ are differential operators of degree $2 r$, which can be written as polynomials in $\partial_{y} \equiv \frac{\partial}{\partial y}$, whose coefficients are polynomial expressions in functions $S_{m}$ and their derivatives (in what follows we denote ${ }^{\prime} \equiv \frac{d}{d x}$ ). The operators $\mathfrak{D}_{r}$ are defined via the generating function

$$
\sum_{r=0}^{\infty} \hbar^{r} \mathfrak{D}_{r}=\exp \left(\sum_{n=1}^{\infty} \hbar^{n} \mathfrak{d}_{n}\right),
$$

where

$$
\mathfrak{d}_{n}=\sum_{r=1}^{n+1} \frac{S_{n+1-r}^{(r)}}{r !}\left(\partial_{y}\right)^{r} .
$$

In particular, for small values of $n$ we get

$$
\begin{aligned}
\mathfrak{d}_{1} & =\frac{1}{2} S_{0}^{\prime \prime} \partial_{y}^{2}+S_{1}^{\prime} \partial_{y}, \\
\mathfrak{d}_{2} & =\frac{1}{6} S_{0}^{\prime \prime \prime} \partial_{y}^{3}+\frac{1}{2} S_{1}^{\prime \prime} \partial_{y}^{2}+S_{2}^{\prime} \partial_{y}, \\
\mathfrak{d}_{3} & =\frac{1}{4 !} S_{0}^{(4)} \partial_{y}^{4}+\frac{1}{3 !} S_{1}^{\prime \prime \prime} \partial_{y}^{3}+\frac{1}{2} S_{2}^{\prime \prime} \partial_{y}^{2}+S_{3}^{\prime} \partial_{y},
\end{aligned}
$$

so that

$$
\begin{aligned}
& \mathfrak{D}_{0}=1 \\
& \mathfrak{D}_{1}=\frac{S_{0}^{\prime \prime}}{2} \partial_{y}^{2}+S_{1}^{\prime} \partial_{y} \\
& \mathfrak{D}_{2}=\frac{\left(S_{0}^{\prime \prime}\right)^{2}}{8} \partial_{y}^{4}+\frac{1}{6}\left(S_{0}^{\prime \prime \prime}+3 S_{0}^{\prime \prime} S_{1}^{\prime}\right) \partial_{y}^{3}+\frac{1}{2}\left(S_{1}^{\prime \prime}+\left(S_{1}^{\prime}\right)^{2}\right) \partial_{y}^{2}+S_{2}^{\prime} \partial_{y},
\end{aligned}
$$

In consequence, at each order $\hbar^{n}$ in (B.4) we get:

$$
\begin{aligned}
\hbar^{0}: & A=0, \\
\hbar^{1}: & \left(\frac{S_{0}^{\prime \prime}}{2} \partial_{y}^{2}+S_{1}^{\prime} \partial_{y}\right) A+A_{1}=0, \\
& \vdots \\
\hbar^{n}: & \mathfrak{D}_{n} A+\mathfrak{D}_{n-1} A_{1}+\ldots+A_{n}=0,
\end{aligned}
$$


We can now use the above formalism to analyze Schrödinger equations for the generalized Catalan and Hurwitz numbers. We already stressed that these equations are special as they have no $\hbar$ corrections, i.e. all $A_{k}=0$ for $k \geq 1$. We can test this statement - or, in other words, reconstruct entire $\widehat{A}$ from the form of $A$ - using (B.8). In particular, from the Proposition 4.4, for Catalan numbers we have

$$
S_{0}^{C}=-\frac{1}{2} z^{2}+\log z, \quad S_{1}^{C}=-\frac{1}{2} \log \left(1-z^{2}\right) .
$$

Plugging into B.7 expressed in terms of $z$ and $\frac{d}{d x}=\left(\frac{d x}{d z}\right)^{-1} \frac{d}{d z}$ (and $x(z)=z+z^{-1}$ ), we indeed find $A_{1}=0$. From the knowledge of the recursion relations for $F_{g, n}$ we could now reconstruct other $S_{m}$ via $(\mathrm{B} .2)$, and use the above hierarchy to show that higher $A_{k}$ vanish as well. However, from our earlier considerations we have essentially proven this, using the form of recursion relations for $F_{g, n}$, without the need of writing down $S_{m}$ explicitly. Nonetheless, in various situations it is necessary to know the form of coefficients $S_{m}$ or the recursion they satisfy, so we can also use the above formalism in this context. The equation (B.8) indeed gives a recursion directly for $S_{m}$, which in addition (due to $A_{k}=0$ for $k \geq 1$ ) reduces simply to $\mathfrak{D}_{n} A=0$. Expressing this recursion in terms of $z$-variable, we find in particular

$$
\begin{aligned}
\frac{d S_{2}^{C}}{d x} & =\frac{z^{5}\left(2 z^{2}+3\right)}{\left(z^{2}-1\right)^{5}} \\
\frac{d S_{3}^{C}}{d x} & =-\frac{5 z^{7}\left(3+7 z^{2}+2 z^{4}\right)}{\left(z^{2}-1\right)^{8}}, \\
\frac{d S_{4}^{C}}{d x} & =\frac{1-9 z^{2}+36 z^{4}-84 z^{6}-4599 z^{8}-13005 z^{1} 0-4440 z^{12}}{360\left(-1+z^{2}\right)^{9}} .
\end{aligned}
$$

Moreover, from $(4.2)$ we have $S_{m}^{C}(z=0)=0$, which fixes a constant of integration, so that integrating the above formulas gives

$$
\begin{aligned}
S_{2}^{C} & =\frac{z^{4}\left(9+z^{2}\right)}{12\left(1-z^{2}\right)^{3}}, \\
S_{3}^{C} & =\frac{5 z^{6}\left(1+z^{2}\right)}{2\left(z^{2}\right)^{6}-1}, \\
S_{4}^{C} & =\frac{z^{8}\left(-4725-12879 z^{2}-4524 z^{4}+36 z^{6}-9 z^{8}+z^{10}\right)}{360\left(z^{2}-1\right)^{9}} .
\end{aligned}
$$

This exercise can also be repeated for the case of the generalized Hurwitz numbers. From (5.14) and (5.20) we have

$$
S_{0}^{H}=z-\frac{1}{2} z^{2}, \quad S_{1}^{H}=-\frac{1}{2}(z+\log (1-z)) .
$$

Plugging into (B.7) confirms that the first correction to the classical Hurwitz curve vanishes, $A_{1}=0$, as it should. Solving further the hierarchy (B.8), which again reduces to $\mathfrak{D}_{n} A=0$, results in

$$
\begin{aligned}
\frac{d S_{2}^{H}}{d x} & =\frac{z^{3}\left(4+z^{2}\right)}{8(1-z)^{5}} \\
\frac{d S_{3}^{H}}{d x} & =-\frac{z^{4}\left(12+8 z+9 z^{2}+z^{4}\right)}{16(z-1)^{8}},
\end{aligned}
$$




$$
\frac{d S_{4}^{H}}{d x}=\frac{z^{5}\left(192+352 z+376 z^{2}+104 z^{3}+76 z^{4}+5 z^{6}\right)}{128(1-z)^{11}} .
$$

These equations can be further integrated to get $S_{m}^{H}$, with the integration constant fixed by $S_{m}^{H}(z=0)=0$, which follows from 5.13 .

Acknowledgement. The authors thank the Banff International Research Center in Alberta and the Hausdorff Research Institute for Mathematics in Bonn for their support and hospitality, where this collaboration was started. They also thank Gaëtan Borot, Vincent Bouchard, Bertrand Eynard, Sergei Gukov, Jerry Kaminker, Maxim Kontsevich, Xiaojun Liu, Marcos Mariño, Michael Penkava, Anne Schilling, Sergey Shadrin, and Don Zagier for useful discussions. The research of M.M. has been supported by NSF DMS-1104734, DMS-1104751, Max-Planck Institut für Mathematik in Bonn, the Beijing International Center for Mathematical Research, American Institute of Mathematics in Palo Alto, the University of Salamanca, and Universiteit van Amsterdam. The research of P.S. has been supported by the DOE grant DE-FG03-92-ER40701FG-02, the European Commission under the Marie-Curie International Outgoing Fellowship Programme, and the Foundation for Polish Science.

\section{REFERENCES}

[1] M. Aganagic, R. Dijkgraaf, A. Klemm, M. Mariño, and C. Vafa, Topological Strings and Integrable Hierarchies, arXiv:hep-th/0312085, Commun. Math. Phys. 261, 451-516 (2006).

[2] M. Aganagic and C. Vafa, Large $N$ duality, mirror symmetry, and a Q-deformed A-polynomial for knots, arXiv:1204.4709v4 [physics.hep-th] (2012).

[3] E. Arbarello, M. Cornalba, and P.A. Griffiths, Geometry of Algebraic Curves, Volume II, Grundlehren der mathematischen Wissenschaften 268, Springer-Verlag 2011.

[4] G. V. Belyi, On galois extensions of a maximal cyclotomic fields, Math. U.S.S.R. Izvestija 14, 247-256 (1980).

[5] G. Borot and B. Eynard, Geometry of spectral curves and all order dispersive integrable system, arXiv:1110.4936 (2011).

[6] G. Borot and B. Eynard, All-order asymptotics of hyperbolic knot invariants from non-perturbative topological recursion of A-polynomials, arXiv:1205.2261 1 [math-ph] (2012).

[7] G. Borot, B. Eynard, M. Mulase and B. Safnuk, Hurwitz numbers, matrix models and topological recursion, Journal of Geometry and Physics 61, 522-540 (2011).

[8] V. Bouchard, A. Klemm, M. Mariño, and S. Pasquetti, Remodeling the B-model, Commun. Math. Phys. 287, 117-178 (2008).

[9] V. Bouchard and M. Mariño, Hurwitz numbers, matrix models and enumerative geometry, Proc. Symposia Pure Math. 78, 263-283 (2008).

[10] A. Brini, B. Eynard, and M. Mariño, Torus knots and mirror symmetry arXiv:1105.2012 (2011).

[11] K. Chapman, M. Mulase, and B. Safnuk, Topological recursion and the Kontsevich constants for the volume of the moduli of curves, Communications in Number theory and Physics 5, 643-698 (2011).

[12] L. Chen, Bouchard-Klemm-Marino-Pasquetti Conjecture for $\mathbb{C}^{3}$, arXiv:0910.3739 (2009).

[13] D. Cooper, D.M. Culler, H. Gillet, D. Long, and P. Shalen, Plane curves associated to character varieties of 3-manifolds, Invent. Math. 118, 47-84 (1994).

[14] R.M. Corless, G.H. Gonnet, D.E.G. Hare, D.J. Jeffrey and D.E. Knuth, On the Lambert W-function, Adv. Computational Math. 5, 329-359 (1996).

[15] R. Dijkgraaf, H. Fuji, and M. Manabe, The volume conjecture, perturbative knot invariants, and recursion relations for topological strings, arXiv:1010.4542 [hep-th] (2010).

[16] R. Dijkgraaf, L. Hollands, and P. Sułkowski, Quantum curves and D-modules, Journal of High Energy Physics 0810.4157, 1-58 (2009).

[17] R. Dijkgraaf, L. Hollands P. Sułkowski, and C. Vafa, Supersymmetric gauge theories, intersecting branes and free Fermions, Journal of High Energy Physics 0802.106, (2008).

[18] R. Dijkgraaf and C. Vafa, Two Dimensional Kodaira-Spencer Theory and Three Dimensional Chern-Simons Gravity, arXiv:0711.1932 [hep-th] (2007).

[19] R. Dijkgraaf, E. Verlinde, and H. Verlinde, Loop equations and Virasoro constraints in non-perturbative twodimensional quantum gravity, Nucl. Phys. B348, 435-456 (1991).

[20] O. Dumitsrescu, M. Mulase, A. Sorkin and B. Safnuk, The spectral curve of the Eynard-Orantin recursion via the Laplace transform, arXiv:1202.1159 [math.AG] (2012). 
[21] P. Dunin-Barkowski, A. Mironov, A. Morozov, A. Sleptsov, and A. Smirnov. Superpolynomials for toric knots from evolution induced by cut-and-join operators, arXiv:1106.4305 [hep-th] (2011).

[22] T. Ekedahl, S. Lando, M. Shapiro, A. Vainshtein, Hurwitz numbers and intersections on moduli spaces of curves, Invent. Math. 146, 297-327 (2001).

[23] B. Eynard, Topological expansion for the 1-hermitian matrix model correlation functions, arXiv:0407261 [hep-th] (2004).

[24] B. Eynard, Recursion between volumes of moduli spaces, arXiv:0706.4403 [math-ph] (2007).

[25] B. Eynard, Intersection numbers of spectral curves, arXiv:1104.0176 (2011).

[26] B. Eynard, M. Mulase and B. Safnuk, The Laplace transform of the cut-and-join equation and the BouchardMariño conjecture on Hurwitz numbers, Publications of the Research Institute for Mathematical Sciences 47, 629-670 (2011).

[27] B. Eynard and N. Orantin, Invariants of algebraic curves and topological expansion, Communications in Number Theory and Physics 1, 347-452 (2007).

[28] B. Eynard and N. Orantin, Weil-Petersson volume of moduli spaces, Mirzakhani's recursion and matrix models, arXiv:0705.3600 [math-ph] (2007).

[29] B. Eynard and N. Orantin, Computation of open Gromov-Witten invariants for toric Calabi-Yau 3-folds by topological recursion, a proof of the BKMP conjecture, arXiv:1205.1103 1 [math-ph] (2012).

[30] J. Fay, Theta functions on Riemann surfaces, Lecture Notes in Mathematics 352, Springer, 1973.

[31] H. Fuji, S. Gukov, and P. Sułkowski, Volume conjecture: refined and categorified, arXiv:1203.2182v1 [hep-th] (2012).

[32] H. Fuji, S. Gukov, and P. Sułkowski, Super-A-polynomial for knots and BPS states, arXiv:1205.1515v2 [hep-th] (2012).

[33] S. Garoufalidis, On the characteristic and deformation varieties of a knot, Geometry \& Topology Monographs 7, 291-309 (2004).

[34] S. Garoufalidis and T.T.Q. Lê, The colored Jones function is qholonomic, Geometry and Topology 9, 1253-1293 (2005).

[35] I.P. Goulden, A differential operato for symmetric functions and the combinatorics of multiplying transpositions, Trans. A.M.S., 344, 421-440 (1994).

[36] I.P. Goulden and D.M. Jackson, Transitive factorisations into transpositions and holomorphic mappings on the sphere, Proc. A.M.S., 125, 51-60 (1997).

[37] T. Graber and R. Vakil, Hodge integrals and Hurwitz numbers via virtual localization, Compositio Math. 135, 25-36 (2003)

[38] S. Gukov, Three-dimensional quantum gravity, Chern-Simons theory, and the A-polynomial, Commun. Math. Phys. 255, 577-627 (2005).

[39] S. Gukov and P. Sułkowski, A-polynomial, B-model, and quantization, arXiv:1108.0002 1 [hep-th] (2011).

[40] J. L. Harer and D. Zagier, The Euler characteristic of the moduli space of curves, Inventiones Mathematicae 85, $457-485$ (1986).

[41] M. Kazarian, KP hierarchy for Hodge integrals, arXiv:0809.3263 (2008).

[42] M. Kazarian, S. Lando, An algebro-geometric proof of Witten's conjecture, J. Amer. Math. Soc. 20, 1079-1089 (2007).

[43] M. Kontsevich, Intersection theory on the moduli space of curves and the matrix Airy function, Communications in Mathematical Physics 147, 1-23 (1992).

[44] M. Kontsevich, Homological algebra of mirror symmetry, arXiv:alg-geom/9411018 (1994).

[45] J. Li, C.-C. M. Liu, K. Liu, and J. Zhou, A mathematical theory of the topological vertex, Geom. Topol. 13 , $527-621(2009)$.

[46] C.-C. M. Liu, Formulae of one-partition and two-partition Hodge Integrals, Geom. \& Top. Monographs 8, 105-128 (2006).

[47] C.-C. M. Liu, Lectures on the ELSV formula, arXiv:1004.0853 In "Transformation Groups and Moduli Spaces of Curves," Adv. Lect. Math. (ALM) 16, 195-216, Higher Education Press and International Press, Beijing-Boston, 2010

[48] C.-C. M. Liu, K. Liu, J. Zhou, A proof of a conjecture of Mariño-Vafa on Hodge Integrals, J. Differential Geom. 65, no. 2, 289-340 (2003).

[49] X. Liu, M. Mulase, and P. Sułkowski, Differential equations and the Eynard-Orantin recursion, in preparation.

[50] M. Mariño, Chern-Simons theory, matrix models, and topological strings, Oxford University Press, 2005.

[51] M. Mariño, Open string amplitudes and large order behavior in topological string theory, J. High Energy Physics 0803-060, 1-33 (2008).

[52] M. Mirzakhani, Simple geodesics and Weil-Petersson volumes of moduli spaces of bordered Riemann surfaces, Invent. Math. 167, 179-222 (2007).

[53] M. Mirzakhani, Weil-Petersson volumes and intersection theory on the moduli space of curves, J. Amer. Math. Soc. 20, 1-23 (2007).

[54] M. Mulase, Algebraic theory of the KP equations, in "Perspectives in Mathematical Physics," R. Penner and S.-T. Yau, Editors, International Press Company, 157-223 (1994). 
[55] M. Mulase, Asymptotic analysis of a Hermitian matrix integral, International Journal of Mathematics 6, 881-892 (1995).

[56] M. Mulase, Lectures on the Asymptotic Expansion of a Hermitian Matrix Integral, in Springer Lecture Notes in Physics 502, H. Aratyn et al., Editors, 91-134 (1998).

[57] M. Mulase and M. Penkava, Ribbon graphs, quadratic differentials on Riemann surfaces, and algebraic curves defined over $\overline{\mathbb{Q}}$, The Asian Journal of Mathematics 2 (4), 875-920 (1998).

[58] M. Mulase and M. Penkava, Topological recursion for the Poincaré polynomial of the combinatorial moduli space of curves, Advances in Mathematics 230, 1322-1339 (2012).

[59] M. Mulase, S. Shadrin, and L. Spitz, The spectral curve and the Schrödinger equation of double Hurwitz numbers and higher spin structures, in preparation.

[60] M. Mulase and N. Zhang, Polynomial recursion formula for linear Hodge integrals, Communications in Number Theory and Physics 4, 267-294 (2010).

[61] M. Mulase and M. Zhou, in preparation.

[62] D. Mumford, Tata lectures on theta II, Jacobian theta functions and differential equations, Progress in Mathematics 43, Birkhäuser 1984 .

[63] P. Norbury, Counting lattice points in the moduli space of curves, arXiv:0801.4590 (2008).

[64] P. Norbury, String and dilaton equations for counting lattice points in the moduli space of curves, arXiv:0905.4141 (2009).

[65] P. Norbury and N. Scott, Polynomials representing Eynard-Orantin invariants, arXiv:1001.0449 (2010).

[66] P. Norbury and N. Scott, Gromov-Witten invariants of $\mathbb{P}^{1}$ and Eynard-Orantin invariants, arXiv:1106.1337 (2011).

[67] A. Okounkov, Toda equations for Hurwitz numbers, Math. Res. Lett. 7, 447 (2000).

[68] A. Okounkov and R. Pandharipande, Gromov-Witten theory, Hurwitz numbers, and matrix models, I, Proc. Symposia Pure Math. 80, 325-414 (2009).

[69] A. Okounkov and R. Pandharipande, Gromov-Witten theory, Hurwitz theory, and completed cycles, Ann. Math. 163, 517-560 (2006).

[70] A. Okounkov and R. Pandharipande, The equivariant Gromov-Witten theory of $\mathbb{P}^{1}$, arXiv:math/0207233 [math.AG] (2002).

[71] H. Ooguri, P. Sulkowski, M. Yamazaki, Wall Crossing As Seen By Matrix Models, arXiv:1005.1293 (2010).

[72] R. Penner, Perturbation series and the moduli space of Riemann surfaces, J. Differ. Geom. 27, 35-53 (1988).

[73] M. Sato, Soliton equations as dynamical systems on an infinite dimensional Grassmannian manifold, Kokyuroku, Res. Inst. Math. Sci., Kyoto Univ. 439, 30-46 (1981).

[74] L. Schneps and P. Lochak, Geometric Galois actions, London Mathematical Society Lecture Notes Series 242 , 1997.

[75] G. Segal and G. Wilson, Loop groups and equations of KdV type, Publ. Math. I.H.E.S., 61, 5-65 (1985).

[76] G. 't Hooft, A planer diagram theory for strong interactions, Nuclear Physics B 72, 461-473 (1974).

[77] R. Vakil, Harvard Thesis 1997.

[78] E. Witten, Two dimensional gravity and intersection theory on moduli space, Surveys in Differential Geometry 1, 243-310 (1991).

[79] J. Zhou, Hodge integrals, Hurwitz numbers, and symmetric groups, preprint, arXiv:math/0308024 [math.AG] (2003).

[80] J. Zhou, On computations of Hurwitz-Hodge integrals, arXiv:0710.1679 (2007).

[81] J. Zhou, Local Mirror Symmetry for One-Legged Topological Vertex, arXiv:0910.4320 (2009).

[82] J. Zhou, Quantum Mirror Curves for $\mathbb{C}^{3}$ and the Resolved Confiold, arXiv:1207.0598 1 [math.AG] (2012).

[83] S. Zhu, The Laplace transform of the cut-and-join equation of Mariño-Vafa formula and its applications arXiv:1001.0618 (2010).

[84] D. Zvonkine, An algebra of power series arising in the intersection theory of moduli spaces of curves and in the enumeration of ramified coverings of the sphere, arXiv:math.AG/0403092 (2004).

Department of Mathematics, University of California, Davis, CA 95616-8633, U.S.A.

E-mail address: mulase@math.ucdavis.edu

Faculty of Physics, University of Warsaw, ul. Hoża 69, 00-681 Warsaw, Poland,

Institute for Theoretical Physics, University of Amsterdam, Science Park 904, 1090 GL, Amsterdam, The Netherlands, AND

Department of Physics, California Institute of Technology, Pasadena, CA 91125, U.S.A.

E-mail address: psulkows@theory.caltech.edu 